\title{
Classical Quantum Field Theory Based on the Hypothesis of the Absolute Reference System
}

\author{
Konstantinos Patrinos \\ National Technical University of Athens, Athens, Greece \\ Email:kpatr@central.ntua.gr
}

How to cite this paper: Patrinos, K. (2019) Classical Quantum Field Theory Based on the Hypothesis of the Absolute Reference System. Journal of Applied Mathematics and Physics, 7, 747-780.

https://doi.org/10.4236/jamp.2019.74052

Received: February 1, 2019

Accepted: March 31, 2019

Published: April 3, 2019

Copyright (c) 2019 by author(s) and Scientific Research Publishing Inc. This work is licensed under the Creative Commons Attribution International License (CC BY 4.0).

http://creativecommons.org/licenses/by/4.0/

\begin{abstract}
The quantum field theory based on the hypothesis of the absolute reference system is a classical non-relativistic theory, which is compatible with current quantum theory. This conclusion arises when one compares the theoretical results of quantum electrodynamics using the basic principles of this hypothesis. Wave equation, which replaces this of Schrodinger, is the classical wave equation of a peculiar electromagnetic wave, derived from the study of particle structure.
\end{abstract}

\section{Keywords}

Particle Mechanics, Field Theory, Quantum Electrodynamics, Quantum Mechanics, Experimental Confirmation of Particle Dynamics

\section{Introduction}

According to the hypothesis of the absolute reference system [1], the wave-behavior of the particles is described by wave functions that are solutions of the classical differential equation of the electromagnetic wave and replace the corresponding solutions of the Schrodinger equation. These wave functions describe real electromagnetic waves originating from the particle photonic structure according to this hypothesis. The states of high energy particles come from the solutions of a modified Dirac equation, which is adapted to the classical perception of this hypothesis. In the first section, two issues of particle dynamics are discussed. One is the Compton effect, and the other is the experiment of Bertozzi, which is one of the historical experiments for confirmation one of the basic principle of the special theory of relativity. In the other sections, a study of the wave-behavior of the particles in quantum mechanics and quantum electrodynamics is discussed. 


\subsection{Compton Effect}

We will examine now the scattering of a photon by an electron, in the reference system of the laboratory (which is the earth's frame of reference), that is Compton effect (ref. [2], paragraph 2.3.4, p. 44, Compton effect), from the point of view of the absolute reference system. We assume that the energy of the photon is $h v$ and the mass of the electron at rest is $m$. At the level $X Y$, the electron momentum vector forms an angle $-\varphi$ with the axis $X$, whereas the direction of the photon forms an angle $\theta$ with the same axis.

Based on what we have mentioned before about the absorption of a high energy photon from a free electron, the phenomenon studied will be accompanied by an increase in the mass of the electron equal to the equivalent mass of a bound photon $m_{p h}=h\left(v-v^{\prime}\right) /\left(2 c^{2}\right)$ (due to the difference in the frequency of the photon incident to the electron and the corresponding outgoing) and also by a kinetic energy absorption equal to:

$$
E_{e}=\frac{1}{4} h\left(v-v^{\prime}\right)
$$

The total energy of the outgoing photon after the impact is equal to $h v^{\prime}$ and the kinetic energy of the electron after the impact, as previously described, is equal to $E_{e}=\left(m+m_{p h}\right) \gamma^{2} u^{2} / 2$. The velocity $u$ is measured with the instruments of the frame of reference of the laboratory and the contraction factor is $\gamma=\left(1-u^{2} / c^{2}\right)^{-1 / 2}$. The momentums of the incident and outgoing photons will be $h v /(2 c)$ and $h v^{\prime} /(2 c)$ respectively; the momentum of the electron is $p$, while the frequency of the deposited mass of the bound photon is equal to $\left(v-v^{\prime}\right) / 2$.

Due to the conservation of momentum on the $X$ axis, the following relation is taken:

$$
\frac{h v}{2 c}=\frac{h v^{\prime}}{2 c} \cos \theta+p \cos \varphi
$$

The conservation of the momentum on the $Y$ axis:

$$
0=\frac{h v^{\prime}}{2 c} \sin \theta-p \sin \varphi
$$

Of these two last relations:

$$
p^{2}=\frac{h^{2}}{4 c^{2}}\left(v-v^{\prime}\right)^{2}+v v^{\prime}(1-\cos \theta) \frac{h^{2}}{2 c^{2}}
$$

According to the previous mentioned and the relation (1.1):

$$
p^{2}=\left(m+m_{p h}\right)^{2} \gamma^{2} u^{2}=\frac{1}{2} m h\left(v-v^{\prime}\right)+\frac{h^{2}\left(v-v^{\prime}\right)^{2}}{4 c^{2}}
$$

From these two latter relations, the change in the wavelength of the photon initially incident to the electron is calculated:

$$
\lambda^{\prime}-\lambda=\frac{c}{v^{\prime}}-\frac{c}{v}=\frac{h}{m c}(1-\cos \theta)
$$




\subsection{The Experiment of W. Bertozzi}

An experiment of controlling the correctness of a proposed dynamics, such as the dynamics of the absolute reference system, is that of $\mathrm{W}$. Bertozzi ${ }^{1}$, which was carried out in the early 1960s, and refers to the measurement of the maximum speed of high energy electrons by a linear accelerator (ref. [4], chapter 1, Departures from Newtonian dynamics, "THE ULTIMATE SPEED”). The already accelerated electrons are released in small bundles (of time duration about $3 \times 10^{-9} \mathrm{sec}$ ), directed to the high-voltage negative end of the Van de Graaff accelerator. The path is described as " 8.4 meter drift space" in Figure 1. Insulated leads at the ends of the path, collect the electrical signals of the beam.

These electrical signals are transmitted on a down-turn oscilloscope via two wires of the same length (so that the signals need equal time to reach the oscilloscope). In this way the pulses displayed on the oscillator give the real time of transmission of the electron beam along the "drift space".

While electron velocity measurements are determined directly using the oscilloscope, kinetic energy is determined from potential difference produced in the Van de Graaff generator and electric field in Linac. This is a strictly predetermined procedure, which has been tested in the laboratory by magnetic deflection methods.

To test any dependency of the electron velocity from the force exerted, due to the very strong electric field, an additional measurement acquired by the high energy electrons is made to a further embodiment comprising an aluminum disk on which impinge the electrons at the end of their path and a thermocouple to measure the temperature increase of the aluminum disc, so the added energy in the form of heat will be proportional to the increase in temperature. In addition, an additional device for measuring the charge collected in the disk is used, in this way to determine the energy transferred from each electron. Such energy measurements were made in the estimated accelerator energies at $1.5 \mathrm{MeV}$ and 4.5 MeV (tested by the above-mentioned magnetic deflection methods), whereby the corresponding values, obtained with the heat increase measurement method in the aluminum disc, were $1.6 \mathrm{MeV}$ and $4.8 \mathrm{MeV}$.

The results of the experiment, listed in Table 1, are five measurements of the electron velocity at corresponding kinetic energy values.

The comparison of experimental results with theoretical calculations of the special theory of relativity and of the hypothesis of the absolute reference system, is certainly the basic criterion of convergence of the experiment with these considerations. The theoretical values of kinetic energy of the special theory of relativity derive from the relation:

$$
E_{\text {rel }}=m(\gamma-1) c^{2}
$$

where $m$ is the mass of the electron, $u$ the electron velocity of the beam and $\gamma=\left(1-u^{2} / c^{2}\right)^{-1 / 2}$. The corresponding theoretical values of the hypothesis of the

${ }^{1}$ The Ultimate Speed, W. Bertozzi, Education Development Center, Newton, Mass. 1962. For more information see reference [3]. 
absolute reference system are taken from the relation:

$$
E_{a b s}=\frac{1}{2} m \gamma^{2} u^{2}
$$

However, the transferred kinetic energy in the target molecules, i.e. the experimentally measured heat, is calculated based on the relative description of the electron collisions of the beam with the atoms of the material. In the absorbtion of a free high energy photon (i.e. of an, elementary, plane electromagnetic wave) from an electron, half of this energy is transferred as kinetic energy, while the other half is available for formation of additional elementary mass. Indeed, in the collision of the beam electrons with the target, the predominant image of the interactions is that of the polarized photons, as an image of elementary plane-waves, that act as interaction photons. Under these conditions the kinetic energy transferred to the target atom, in the form of heat, will be equal to:

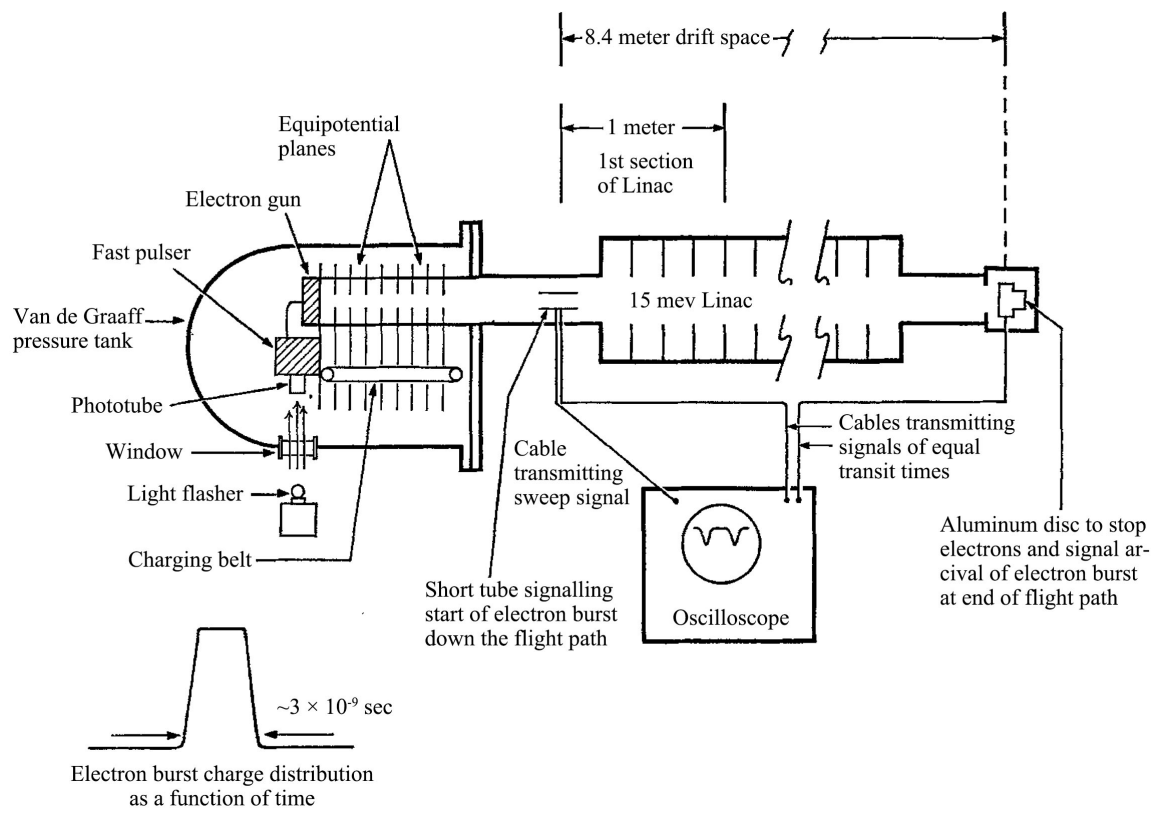

Figure 1. The apparatus schematic diagram of measuring of the electron experimental flight time and of the electrons energy. The electrons have already accelerated due to the existence of a strong electric field of the Van de Graaff generator.

Table 1. Experimental results of W. Bertozzi's measurements, as set out in his work entitled “The Ultimate Speed”, in 1964.

\begin{tabular}{cccc}
\hline kinetic & flight & electron & \\
\hline energy & time & velocity & \\
$K, \mathrm{MeV}$ & $t, \times 10^{-8} \mathrm{sec}$ & $u, \times 10^{8} \mathrm{~m} / \mathrm{sec}$ & $u^{2}, \times 10^{16} \mathrm{~m}^{2} / \mathrm{sec}^{2}$ \\
0.5 & 3.23 & 2.60 & 6.8 \\
1.0 & 3.08 & 2.73 & 7.5 \\
1.5 & 2.92 & 2.88 & 8.3 \\
4.5 & 2.84 & 2.96 & 8.8 \\
15 & 2.80 & 3.00 & 9.0 \\
\hline
\end{tabular}




$$
E_{a b s}=\frac{1}{4} m \gamma^{2} u^{2}
$$

In an intermediate state, where the electrons of the beam move at speeds that are not very close to the velocity of light in the vacuum, a part of the total number of force carriers will transfer the total kinetic energy to the target atoms, while the remaining force carriers the half of the kinetic energy. In this case, the energy transferred in the form of heat to the target atom will have a value between $(1 / 4) m \gamma^{2} u^{2}$ and $(1 / 2) m \gamma^{2} u^{2}$.

Table 2 includes in the first column the ratio of speeds $u / c$, in the second column the experimental values of the heat of the target, while in the third and fourth column the corresponding theoretical values derived from hypothesis of the absolute system and the special theory of relativity respectively.

The values of the first two lines in the table correspond to speeds equals to $87 \%$ and $91 \%$ of the light velocity in the vacuum, not so close to $100 \%$. Since, according to the hypothesis of the absolute reference system, the heat transferred to the target has values between (1/4) $m \gamma^{2} u^{2}$ and (1/2) $m \gamma^{2} u^{2}$, corresponding values are those listed in Table 2. Therefore, the experimental values are indeed within those ranges. The values of the last two lines of the table correspond to velocities very close to the speed of light in the vacuum, and according to the above, the corresponding calculated atomic heat is equal to $(1 / 4) m \gamma^{2} u^{2}$. These high energy values are, as shown in Table 2, equal to $1.48 \mathrm{MeV}$ and $4.67 \mathrm{MeV}$, with a small difference from the corresponding experimental ones. This is a confirmation of the hypothesis of the absolute system ${ }^{2}$.

The theoretical values derived from the special theory of relativity are confirmed only in the first line of the table, while the rest are much smaller than the corresponding experimental ones. These values might be somewhat acceptable if they were larger than the corresponding experimental ones. Therefore the experimental results are in accordance with the special theory of relativity only in that the higher speed in nature is that of the velocity of light in the vacuum. However, it is not in agreement with the corresponding experimental values of energy.

Table 2. Table of experimental and theoretical values of the special theory of relativity and absolute reference system.

\begin{tabular}{cccc}
\hline $\mathrm{u} / \mathrm{c}$ & Experimental & values of energy & values of energy \\
\hline ratio & values of energy & of the absolute system & of special relativity \\
0.87 & $(\mathrm{MeV})$ & $(\mathrm{MeV})$ & $(\mathrm{MeV})$ \\
0.91 & 0.5 & $0.39-0.78$ & 0.52 \\
0.96 & 1.0 & $0.61-1.22$ & 0.72 \\
0.99 & 1.5 & 1.48 & 1.30 \\
\hline
\end{tabular}

${ }^{2}$ About experimental confirmation of particle dynamics from the point of view of the hypothesis of absolute reference system see reference [5], sections 1.3, 2.4 and 3.9. 


\section{The Wave Behavior of Particles}

If photons are the structural component of matter, then a self-evident conclusion is that particle movements should obey a wave equation similar to the differential equation of the electromagnetic wave. It seems, according to the relative theoretical analysis of this section, in the light of the hypothesis of the absolute reference system, that the theoretical results of this assumption are acceptable, since they are in accordance with those of modern physics.

\subsection{Particle-Frequency and Wavelength}

A particle, as previously described, is composed of a number of bound photons and its total energy will be determined as the sum of total energies of these photons. The total energy derived from the mass frequencies of all the bound photons of the particle shall be equal to:

$$
E_{0}=\frac{1}{2} m c^{2}=\frac{1}{2} h \sum_{i=1}^{N} v_{c_{i}}
$$

where $v_{c_{i}}$ is the mass frequency of the $i$ bound photon, $\sum_{i=1}^{N} v_{c_{i}}$ is the sum of all mass frequencies and $N$ is the number of bound photons. This latter equation results from the relation $(1 / 2) h v_{c_{i}}=(1 / 2) m_{p h h_{i}} c^{2}$, so $\sum_{i=1}^{N} v_{c_{i}}=\sum_{i=1}^{N} m_{p h_{i}} c^{2} / h=m c^{2} / h$. We also accept, especially for very small particles (e.g., electrons), that the mass frequencies of the bound photons (which are similar to energy levels of atomic electrons) are different each other. The total energy of the particle will be:

$$
E_{t o t}=\frac{1}{2} m \gamma^{2} c^{2}=\frac{1}{2} h \sum_{i=1}^{N}\left(v_{c_{i}}+v_{u_{i}}\right)
$$

where $\gamma=\left(1-u^{2} / c^{2}\right)^{-1 / 2}$ and $u$ is the velocity of the particle measured by the measuring instruments of the laboratory inertial frame. We also assume that the total frequency of the $i$ bound photon is equal to $v_{c_{i}}+v_{u_{i}}$, where $v_{u_{i}}$ is the transfer frequency of the $i$ bound photon.

The kinetic energy of the particle will result from the difference:

$$
E_{\text {kin }}=E_{\text {tot }}-E_{0}=\frac{1}{2} m \gamma^{2} u^{2}=\frac{1}{2} h \sum_{i=1}^{N} v_{u_{i}}
$$

We define the quantity $v_{q}=\sum_{i=1}^{N} v_{u_{i}}$ as particle transfer frequency or simply as a particle frequency. Accordingly, the transfer frequency of the $i$ bound photon will be equal to the amount $v_{u_{i}}=m_{p h_{i}} \gamma^{2} u^{2} / h$, and since $m_{p h_{i}}=h v_{c_{i}} / c^{2}$, the frequencies ratio is:

$$
\frac{v_{u_{i}}}{v_{c_{i}}}=\frac{\gamma^{2} u^{2}}{c^{2}}
$$

Since the mass frequencies of the bound photons are different from each other, the transfer frequencies of all the bound photons, based on the latter relation, will be different from each other. Therefore, the sum of the transfer frequencies of all bound photons will be equal to the particle frequency. This frequency (which is actually a sum of frequencies) accompanies the movement of each par- 
ticle and is a characteristic of its reference system.

Therefore the kinetic energy in relation to the particle frequency is:

$$
E_{k i n}=\frac{1}{2} m \gamma^{2} u^{2}=\frac{1}{2} h v_{q}
$$

Also the corresponding particle wavelength $\lambda$ is determined by the relation $\gamma u=v_{q} \lambda$. Therefore, the momentum of the particle is:

$$
p=\hbar k
$$

where $k$ (the wavenumber) is equal to $2 \pi / \lambda$.

\subsection{Wave Function of a Free Particle}

The wave function $\Psi(\boldsymbol{r}, t)$ of a free electron must obey, according to the absolute system hypothesis, not in the Schrodinger equation but in the differential equation of an peculiar electromagnetic wave:

$$
\nabla^{2} \Psi(\boldsymbol{r}, t)-\frac{1}{\gamma^{2} u^{2}} \frac{\partial^{2} \Psi(\boldsymbol{r}, t)}{\partial t^{2}}=0
$$

which propagates at a velocity $\gamma u$, and not at the velocity of propagation of the light in the vacuum.

This wave function as a solution of the wave equation will be of the form ${ }^{3}$ :

$$
\Psi(\boldsymbol{r}, t)=A \mathrm{e}^{i\left(\boldsymbol{r} \cdot \boldsymbol{k}-2 \pi v_{q} t\right)}=A \mathrm{e}^{\frac{i}{\hbar}\left(\boldsymbol{p} \cdot \boldsymbol{r}-2 E_{k i n} t\right)}
$$

where the constant $A$ is the amplitude of the particle wave, $E_{k i n}=(1 / 2) m \gamma^{2} u^{2}$, $p=m_{p h} \gamma u$ and $\gamma=\left(1-u^{2} / c^{2}\right)^{-1 / 2}$. If we define the operators of momentum and energy with relations:

$$
\begin{gathered}
\hat{p}=-i \hbar \nabla \\
\hat{E}_{k i n}=\frac{i \hbar}{2} \frac{\partial}{\partial t}
\end{gathered}
$$

the equation of momentum in relation to energy for a free particle $p=2 E_{k i n} /(\gamma u)$ is equivalent to the equation:

$$
\hat{p}^{2} \Psi(\boldsymbol{r}, t)=\frac{1}{\gamma^{2} u^{2}}\left(2 \hat{E}_{k i n}\right)^{2} \Psi(\boldsymbol{r}, t)
$$

which is the aforementioned differential wave equation.

The operator of momentum is the same as that of the Schrodinger quantum mechanics, but the operator of energy is differentiated by a factor equal to $1 / 2$, since the operator of energy of the Schrodinger quantum mechanics is equal to $\hat{E}=i \hbar \frac{\partial}{\partial t}$.

If the constant $A$ of the solution of the differential equation of electromagnetic wave is the electric field amplitude derived from the sum of all the electric fields of all the elemental photonic electromagnetic waves accompanying the move-

${ }^{3}$ ref. [6], Chapter 7, Plane Electromagnetic Waves and Wave Propagation, section 7.1, Plane Waves in a Nonconducting Medium. 
ment of the particle, then the quantity $A^{2}$ is proportional to the energy density of these electric fields, and also it is proportional to the density of the mass inside the particle-space. Since the amount of this energy is constant for each particle, in a parallel beam of same particles with the same number of bound photons per particle, the magnitude $\int_{V_{0}}|\Psi|^{2} \mathrm{~d} V$ will be proportional to the number of particles in the volume $V_{0}$.

The particle current corresponding to the wave function $\Psi(r, t)$ will result from the free particle wave Equation (2.7). By multiplying both members of this equation with $\Psi^{*}$ and also writing the conjugate expression, we have the relations:

$$
\begin{aligned}
& \frac{1}{\gamma^{2} u^{2}} \Psi^{*} \frac{\partial^{2} \Psi}{\partial t^{2}}=\Psi^{*} \nabla^{2} \Psi \\
& \frac{1}{\gamma^{2} u^{2}} \Psi \frac{\partial^{2} \Psi^{*}}{\partial t^{2}}=\Psi \nabla^{2} \Psi^{*}
\end{aligned}
$$

and therefore:

$$
\frac{\partial}{\partial t}\left(\frac{i \hbar}{2 m \gamma^{2} u^{2}}\left(\Psi^{*} \frac{\partial \Psi}{\partial t}-\Psi \frac{\partial \Psi^{*}}{\partial t}\right)\right)=-\nabla\left(\frac{i \hbar}{2 m} \Psi \nabla \Psi^{*}-\Psi^{*} \nabla \Psi\right)
$$

so, based on the continuity equation $\frac{\partial \rho}{\partial t}+\nabla \cdot \boldsymbol{j}=0$, we have got:

$$
\begin{gathered}
\rho=\frac{i \hbar}{2 m \gamma^{2} u^{2}}\left(\Psi^{*} \frac{\partial \Psi}{\partial t}-\Psi \frac{\partial \Psi^{*}}{\partial t}\right) \\
\boldsymbol{j}=\frac{i \hbar}{2 m}\left(\Psi \nabla \Psi^{*}-\Psi^{*} \nabla \Psi\right)
\end{gathered}
$$

According to relations $\hat{E}_{k i n} \Psi=(i \hbar / 2) \partial \Psi / \partial t=E_{k i n} \Psi, \hat{p} \Psi=-i \hbar \nabla \Psi=\boldsymbol{p} \Psi$, $E_{k i n}=(1 / 2) m \gamma^{2} u^{2}, \quad \boldsymbol{p}=m \gamma \boldsymbol{u}$, for low speeds the following relations arise:

$$
\begin{gathered}
\rho=\Psi \Psi^{*} \\
\boldsymbol{j}=\frac{\boldsymbol{p}}{m} \Psi \Psi^{*}=\rho \gamma \boldsymbol{u}
\end{gathered}
$$

When the particle moves within a potential, then as we shall see later, the solution of the differential equation will surely vary as compared to that of the free particle. In this case, for a plurality of same particles, under the influence of this potential, the quantity $\int_{V_{0}}|\Psi|^{2} \mathrm{~d} V$ will also be proportional to the number of particles in the volume $V_{0}$. For one particle the quantity $|\psi(\boldsymbol{r})|^{2}$ is proportional to the density of mass on the location $r$ of the particle-space. Therefore, the wave function $\Psi(\boldsymbol{r}, t)$ has a different interpretation from that of the corresponding Schrodinger wave function, since it is classical and is a solution not of the Schrodinger equation but of the above-mentioned differential equation of electromagnetic wave.

As we will see in Section 4, on quantum field theory based on the hypothesis of the absolute reference system, the Klein-Gordon equation and the Dirac equ- 
ation, which are used in the case where the velocity of the particle is comparable to the velocity of light in the vacuum, are acceptable by the hypothesis of the absolute reference system. All the theoretical results of quantum field theory (for example, the theoretical results of quantum electrodynamics) are accepted by the hypothesis of the absolute system, if interpreted in the basis of this theory.

\subsection{Wave Function of a Particle in the Presence of an External Potential}

Starting from the assumption that an initially free charged particle, for example a free electron or a parallel electron beam, enters a space with stable electrical potential, with some initial conditions of the problem, we reach a differential equation. The solution of this equation gives the ability to determine the number of particles, as a function of the location. If the dynamic energy $V$ of an electron is positive but less than the initial kinetic energy $T$, then the kinetic energy in the space of existing potential is $T_{p}=T-V$ and the wave differential equation is described in the previous subsection. When the dynamic energy is greater than the initial kinetic energy, then the kinetic energy $T_{p}$ is negative, while the corresponding momentum value $p=\sqrt{2 m T_{p}}$ is imaginary and the corresponding solutions of the wave equation are exponential functions. Relative examples are those of the subsections 3.5 and 3.6. This is indeed a mathematical representation of a natural absorption phenomenon such as the propagation of an electromagnetic wave into a conductor (reference [7], CHAPTER XIII, OPTICS OF METALS, paragraph 13.1, WAVE PROPAGATION IN A CONDUCTOR), where the values of wavelength and refractive index are complex. This whole image shows a particle wave behavior similar to that of reflection, refraction, or absorption of an electromagnetic wave, that is, a beam of free photons incident to a material that may be reflective, transparent or absorbent.

The equations to which it generally obeys a particle motion to an external potential $V$ is the energy conservation equation $E=T+V$ and the differential equation of the particle wave. This differential wave-equation results from the equation of kinetic energy determination $E-V=T=p^{2} /(2 m)$, making use of the operator $\hat{p}=-i \hbar \nabla$, as in the previous subsection. The corresponding differential equation, in the case of a particle whose total energy is constant (time-independent), is the same as that of Schrodinger:

$$
\frac{-\hbar^{2}}{2 m} \nabla^{2} \Psi(\boldsymbol{r})-(E-V(\boldsymbol{r})) \Psi(\boldsymbol{r})=0
$$

By including the time evolution of this particle's state, we arrive at a more general form of the wave function, $\Psi(\boldsymbol{r}, t)=\Psi(\boldsymbol{r}) \mathrm{e}^{i\left(2 \pi v_{q} t\right)}$, where $2 \pi v_{q}=\omega=2 T / \hbar$. This wave function satisfies the last differential equation, but also that which results from the relation $p^{2}=\left(1 /\left(\gamma^{2} u^{2}\right)\right)(2 T)^{2}$ and from the operator $\hat{T}=(i \hbar / 2)(\partial / \partial t)$ and is the following:

$$
\frac{-\hbar^{2}}{2 m \gamma^{2} u^{2}} \frac{\partial^{2}}{\partial t^{2}} \Psi(\boldsymbol{r}, t)-(E-V(\boldsymbol{r})) \Psi(\boldsymbol{r}, t)=0
$$


It also, of course, satisfies the wave-equation:

$$
\nabla^{2} \Psi(\boldsymbol{r}, t)-\frac{1}{\gamma^{2} u^{2}} \frac{\partial^{2}}{\partial t^{2}} \Psi(\boldsymbol{r}, t)=0
$$

\subsection{Particle Motion in Closed Orbits}

For an electron moving in an atomic scale space, under the influence of the Coulomb field, the corresponding force exerted on it will have values that correspond to the same scale (that is, this force can not be enormous), and therefore, its velocity will not be comparable to the speed of light in the vacuum. So, the calculated contraction factor value is very close to $1(\gamma \simeq 1)$. Such examples that will be studied in the next section are those of an electron in a hydrogen atom, the example of the potential of harmonic oscillator, and the example of an electron moving periodically inside an infinite potential well.

According to mentioned in the previous subsection 2.1 about the kinetic energy in relation to particle frequency, according to the relation (2.5) presented in subsection 2.1 the frequency of the particle is proportional to kinetic energy. Therefore, in a closed periodic motion of an electron, the average value of the kinetic energy, over a time period equal to that required for a complete closed orbit of the particle, will be proportional to the average value of this frequency. This results in the following relation:

$$
\overline{E_{\text {kin }}}=\frac{1}{2} m \overline{u^{2}}=\frac{1}{2} h \overline{v_{q}}
$$

where $\overline{E_{k i n}}, \overline{u^{2}}, \overline{v_{q}}$ are time average values of kinetic energy, of velocity squared, and of particle frequency respectively. A particular definition, which could be used in the consideration of wave motion of the electron, is that of an "equivalent particle-wavelength" according to the relation:

$$
\lambda_{q}=\frac{\sqrt{\overline{u^{2}}}}{\overline{v_{q}}}
$$

and respectively is defined as "equivalent time period of oscillation" of the particle-wave the amount $T_{q}$, calculated as following :

$$
T_{q}=\frac{1}{\overline{v_{q}}}
$$

Since the electron must behave, based on its structure, like a wave, in order for the movement of its closed orbit to be stable, this wave should be a stationary wave. Therefore, an additional binding condition is introduced which governs the periodic motion under consideration and is that the length of the closed orbit should be an integer multiple of the equivalent particle-wavelength.

If the $\ell_{n}$ length of the closed orbit is equal to $n \lambda_{q}$, where $n$ is an integer, then the time $T_{n}$ of a closed path is equal to $n T_{q}$ and therefore the frequency $v_{n}=1 / T_{n}$ is related to the corresponding mean particle frequency:

$$
\overline{v_{q}}=n v_{n}
$$


and finally a general equation is:

$$
\overline{E_{k i n}}=\frac{1}{2} m \overline{u^{2}}=\frac{1}{2} h n v_{n}
$$

This result, taking into account the equation of motion of the electron, according to the relative examples exposed in the next section, leads to the conclusion that the energy of the electron and also all physical quantities involved in this problem are quantized.

\subsection{Uncertainty Principle}

In the subsection 2.2 it is stated that the quantity $|\psi(\boldsymbol{r})|^{2}$ is proportional to the density of the mass in the space of the particle. This density is proportional to the number of bound photons per unit volume. Because a particle is located in a small area, rather than a single point, the location of the particle can be considered as the point where the density is maximized. While the density distribution of the particle mass appears to be continuous according to the function $|\psi(\boldsymbol{r})|^{2}$, it is distinct since the particle consists of a number of bound photons. The limits up to which the particle mass extends are those points in which, in a volume $\delta V$ equal to the volume of the smallest bound photon, there is a calculated mass (proportional to the amount of $\left.\int_{\delta V}|\psi(\boldsymbol{r})|^{2} \mathrm{~d} V\right)$ smaller than the mass of the same bound photon.

By normalizing the function $\psi(\boldsymbol{r})$ so that the integral in the infinite space is equal to the unit according to the relation:

$$
\int_{-\infty}^{\infty} \int_{-\infty}^{\infty} \int_{-\infty}^{\infty}|\psi(\boldsymbol{r})|^{2} \mathrm{~d}^{3} \boldsymbol{r}=1
$$

the amount $\int_{x_{1}}^{x_{2}} \int_{y_{1}}^{y_{2}} \int_{z_{1}}^{z_{2}}|\psi(\boldsymbol{r})|^{2} \mathrm{~d} x \mathrm{~d} y \mathrm{~d} z$ is the fraction of the unit which is equal to the ratio $\delta m / m$, where $\delta m$ is the mass inside the volume $\int_{x_{1}}^{x_{2}} \int_{y_{1}}^{y_{2}} \int_{z_{1}}^{z_{2}} \mathrm{~d} x \mathrm{~d} y \mathrm{~d} z$ and $m$ is the mass of the particle.

The mathematical development of the subject here was done by Andre Kessler. ${ }^{4}$ It turns out that the Fourier conjugate of a very localized waveform will be spread out. Thus, if position and momentum (or energy and time, etc.) are Fourier conjugates, and if you know the position to a high degree of accuracy, then you don't know the momentum very well and vice versa.

If $\psi(\boldsymbol{r})$ is the spatial part of the wave function, then this can be written in the form of a Fourier transform as follows:

$$
\psi(\boldsymbol{r})=\frac{1}{(\sqrt{2 \pi})^{3}} \int_{-\infty}^{\infty} \int_{-\infty}^{\infty} \int_{-\infty}^{\infty} \Psi(\boldsymbol{k}) \mathrm{e}^{i \boldsymbol{k} \cdot \boldsymbol{r}} \mathrm{d}^{3} \boldsymbol{r}
$$

The inverse Fourier transform is:

$$
\Psi(\boldsymbol{k})=\frac{1}{(\sqrt{2 \pi})^{3}} \int_{-\infty}^{\infty} \int_{-\infty}^{\infty} \int_{-\infty}^{\infty} \psi(\boldsymbol{r}) \mathrm{e}^{-i \boldsymbol{k} \cdot \boldsymbol{r}} \mathrm{d}^{3} \boldsymbol{k}
$$

4"Derivation of the Heisenberg Uncertainty Principle", Andre Kessler, Department of Mathematics, Massachusetts Institute of Technology. 
The momentum will be given by the relation $\boldsymbol{p}=\hbar \boldsymbol{k}$. We should expect the classical momentum to be the average value, and other values to be less probable. The corresponding probability will be expressed with a normal distribution. This implies that:

$$
P_{k}=A_{k} \mathrm{e}^{-\left(\boldsymbol{k}-\boldsymbol{k}_{0}\right)^{2} /\left(2 \sigma_{k}^{2}\right)}
$$

where $\boldsymbol{k}_{0}$ the average value of wave number $\boldsymbol{k}$, and $\sigma_{\boldsymbol{k}}$ is the standard deviation. Therefore:

$$
\Psi(\boldsymbol{k})=\sqrt{P_{\boldsymbol{k}}}=\sqrt{A_{\boldsymbol{k}}} \mathrm{e}^{-\left(\boldsymbol{k}-\boldsymbol{k}_{0}\right)^{2} /\left(4 \sigma_{\boldsymbol{k}}^{2}\right)}
$$

Like earlier, we should expect the classical position to be the average value, and other values to be less probable, and therefore the probability of position is expressed by a normal distribution. If we let $\boldsymbol{r}_{0}$ be the most likely position for the particle, then a normal distribution of the positions is:

$$
P_{r}=A_{r} \mathrm{e}^{-\left(\boldsymbol{r}-\boldsymbol{r}_{0}\right)^{2} /\left(2 \sigma_{r}^{2}\right)}
$$

where $\boldsymbol{r}_{0}$ is the average position value, and $\sigma_{r}$ is the corresponding standard deviation.

Since the function $P_{r}$ is the upper envelope of the function $|\psi(\boldsymbol{r})|^{2}$, the envelope of the function $\psi(\boldsymbol{r})$ is:

$$
\psi(\boldsymbol{r})_{e n v}=\sqrt{P_{r}}=\sqrt{A_{r}} \mathrm{e}^{-\left(\boldsymbol{r}-\boldsymbol{r}_{0}\right)^{2} /\left(4 \sigma_{\boldsymbol{r}}^{2}\right)}
$$

The coefficients $A_{r}$ and $A_{k}$ are calculated by simply normalizing the normal distribution:

$$
\int_{-\infty}^{\infty} \int_{-\infty}^{\infty} \int_{-\infty}^{\infty} P_{\boldsymbol{r}} \mathrm{d}^{3} \boldsymbol{r}=\int_{-\infty}^{\infty} \int_{-\infty}^{\infty} \int_{-\infty}^{\infty} P_{\boldsymbol{k}} \mathrm{d}^{3} \boldsymbol{k}=1
$$

this gets us:

$$
A_{r}=\frac{1}{\left(\sigma_{r} \sqrt{2 \pi}\right)^{3}}, \quad A_{k}=\frac{1}{\left(\sigma_{k} \sqrt{2 \pi}\right)^{3}}
$$

Since the $\psi(\boldsymbol{r})_{e n v}$ and $\Psi(\boldsymbol{k})$ functions are finally functions of $\boldsymbol{r}-\boldsymbol{r}_{0}$ and $\boldsymbol{k}-\boldsymbol{k}_{0}$ respectively the Fourier transforms can be rewritten as follows:

$$
\begin{gathered}
\psi(\boldsymbol{r})_{e n v}=\frac{1}{(\sqrt{2 \pi})^{3}} \int_{-\infty}^{\infty} \int_{-\infty}^{\infty} \int_{-\infty}^{\infty} \Psi(\boldsymbol{k}) \mathrm{e}^{i\left(\boldsymbol{k}-\boldsymbol{k}_{0}\right) \cdot\left(\boldsymbol{r}-\boldsymbol{r}_{0}\right)} \mathrm{d}^{3} \boldsymbol{k} \\
\Psi(\boldsymbol{k})=\frac{1}{(\sqrt{2 \pi})^{3}} \int_{-\infty}^{\infty} \int_{-\infty}^{\infty} \int_{-\infty}^{\infty} \psi(\boldsymbol{r})_{e n v} \mathrm{e}^{-i\left(\boldsymbol{k}-\boldsymbol{k}_{0}\right) \cdot\left(\boldsymbol{r}-\boldsymbol{r}_{0}\right)} \mathrm{d}^{3} \boldsymbol{r}
\end{gathered}
$$

After the integration of the second member of the last equation, the following equation is taken:

$$
\frac{1}{\left(\sqrt{\sigma_{k} \sqrt{2 \pi}}\right)^{3}} \mathrm{e}^{-\left(\boldsymbol{k}-\boldsymbol{k}_{0}\right)^{2} /\left(4 \sigma_{k}^{2}\right)}=\frac{1}{(\sqrt{2 \pi})^{3}}\left(\frac{\sqrt{2} \sigma_{r} \sqrt{2 \pi}}{\sqrt{\sigma_{r} \sqrt{2 \pi}}}\right)^{3} \mathrm{e}^{-\left(\boldsymbol{k}-\boldsymbol{k}_{0}\right)^{2} \sigma_{r}^{2}}
$$


In the last equation, the coefficients and exhibitors must be equal. The two equations:

$$
\left(\frac{1}{\sigma_{k} \sqrt{2 \pi}}\right)^{3 / 2}=\left(\frac{2 \sigma_{r}}{\sqrt{2 \pi}}\right)^{3 / 2},-\left(\boldsymbol{k}-\boldsymbol{k}_{0}\right)^{2} /\left(4 \sigma_{k}^{2}\right)=-\left(\boldsymbol{k}-\boldsymbol{k}_{0}\right)^{2} \sigma_{r}^{2}
$$

end up in exactly the same relation:

$$
\sigma_{k} \sigma_{r}=\frac{1}{2}
$$

Due to the relation $\boldsymbol{k}=\boldsymbol{p} / \hbar$, the standard deviation of the position in relation to the standard deviation of the momentum is $\sigma_{r}=\sigma_{p} / \hbar$. Therefore:

$$
\sigma_{p} \sigma_{r}=\frac{\hbar}{2}
$$

This, of course, the latter is true only if the probability distribution is normal. If it isn't $\sigma_{p} \sigma_{r}$ will be greater, as the normal distribution turns out to have the minimum possible product. Therefore, in the general case:

$$
\sigma_{p} \sigma_{r} \geq \frac{\hbar}{2}
$$

or else:

$$
\Delta p \Delta r \geq \frac{\hbar}{2}
$$

This is the Heisenberg uncertainty principle for position and momentum.

The time-dependent part of wave function $\psi(t)$ can be written in the form of a Fourier transform as follows:

$$
\psi(t)=\frac{1}{\sqrt{2 \pi}} \int_{-\infty}^{\infty} \Psi(\omega) \mathrm{e}^{i \omega t} \mathrm{~d} \omega
$$

The inverse Fourier transform can be written as follows:

$$
\Psi(\omega)=\frac{1}{\sqrt{2 \pi}} \int_{-\infty}^{\infty} \psi(t) \mathrm{e}^{-i \omega t} \mathrm{~d} t
$$

here the quantity $\omega /(2 \pi)$ is considered equal to the particle frequency $v_{q}$, as defined in the previous subsections. By following the same process as that of the spatial part of the wave function, using a normal distribution of frequencies and times, we arrive at the following relation:

$$
\sigma_{\omega} \sigma_{t}=\frac{1}{2}
$$

where $\omega=2 \pi v_{q}$. Also kinetic energy is given by relation $E_{\text {kin }}=(1 / 2) \hbar \omega$. So, the last relation can be written as follows:

$$
\Delta E_{\text {kin }} \Delta t=\frac{\hbar}{4}
$$

As above, in the general case:

$$
\Delta E_{k i n} \Delta t \geq \frac{\hbar}{4}
$$


In the case of a harmonic oscillator the total energy is equal to $E=\hbar \omega$ and therefore:

$$
\Delta E \Delta t \geq \frac{\hbar}{2}
$$

This last relation is the Heisenberg uncertainty principle for energy and time.

\section{Examples of Electron Motion in Various Potentials}

We will then look at some of the best-known examples of quantum mechanics in the light of the hypothesis of the absolute reference system. The results obtained by solving these examples seem to be fully in agreement with the corresponding results of Schrodinger's quantum mechanics.

If we consider as initial condition the relation $\gamma \simeq 1$, and $\gamma$ is denoted by the contraction factor of the particle's reference system, then the expressions for the energy, momentum, etc, are that of Newtonian physics. Under this condition, the equations of the motions and the energies of electrons, in a good approach, are those of Newtonian mechanics.

\subsection{Circular Motion of Electron in Coulomb Potential}

A simplified example of an electron's motion in a Coulomb field is that of circular motion. In this case the field comes from a unique proton. In the general case, such as the Hydrogen atom, the orbit of the electron is elliptical, but this subject will be examined in a next example.

The total energy of the electron in our example is:

$$
E=\frac{1}{2} m u^{2}-\frac{e^{2}}{r}
$$

where $m$ is the mass, $u$ is the electron velocity, $e$ is the elementary charge, and $r$ is the position of the electron in a cartesian coordinate system, with origin the center of mass of the proton-electron system. Based on the centripetal force exerted on the electron $F_{c}=m u^{2} / r=e^{2} / r^{2}$, the total energy is:

$$
E=-\frac{1}{2} m u^{2}
$$

Since the speed remains constant, based on the relation (2.23) presented in subsection 2.4, the average value of velocity-squared is $\overline{u^{2}}=u_{n}^{2}$, and the kinetic energy is:

$$
E_{k i n, n}=\frac{1}{2} m u_{n}^{2}=\frac{1}{2} \frac{e^{2}}{r_{n}}=\frac{1}{2} h n v_{n}
$$

The frequency $v_{n}$ due to its definition will be the frequency of the circular motion of the electron (that is, the number of rotations in the time unit), so, the velocity is given by the relation:

$$
u_{n}=2 \pi v_{n} r_{n}
$$

and therefore on the basis of the foregoing, the total energy, speed, the radius of 
the circular track and the angular momentum, are given by the following relations:

$$
\begin{gathered}
E_{n}=-\frac{m e^{4}}{2 \hbar^{2}} \frac{1}{n^{2}} \\
u_{n}=\frac{e^{2}}{\hbar} \frac{1}{n} \\
r_{n}=\frac{\hbar^{2}}{m e^{2}} n^{2} \\
L_{n}=n \hbar
\end{gathered}
$$

In the same relations one ends up, applying Bohr's theory of circular motion.

\subsection{Infinite Potential Well}

We assume that the potential at the $X$ axis is zero in the range $0<x<L$ and infinite outside this range. An electron moves in the direction of the $X$ axis, with a velocity $u$ in the range $0<x<L$. When it impinges on the infinite potential walls, its velocity is reversed. So, the motion of the electron is periodic. The orbit of the electron is closed, and in a period $T_{n}$ it traverses a length $2 L$.

The kinetic energy of the electron is:

$$
E=\frac{1}{2} m u^{2}
$$

where $m$ is the mass of the electron. Since the speed remains constant, based on the relation (2.23) presented in subsection 2.4, the average of the velocity-squared is $\overline{u^{2}}=u_{n}^{2}$, and the kinetic energy is:

$$
E_{k i n, n}=\frac{1}{2} m u_{n}^{2}=\frac{1}{2} h n v_{n}
$$

The speed is equal to $u_{n}=2 L v_{n}$ (the $n$ is always integer), so:

$$
v_{n}=\frac{h}{4 m L^{2}} n
$$

The kinetic energy is:

$$
E_{n}=\frac{\pi^{2} \hbar^{2}}{2 m L^{2}} n^{2}
$$

The momentum is:

$$
p_{n}=m u_{n}=\frac{\pi \hbar}{L} n
$$

\subsection{Harmonic Oscillator}

The potential of the one-dimensional harmonic oscillator, in the $\mathrm{X}$ direction, is given by the parabolic form:

$$
V(x)=\frac{1}{2} k x^{2}=\frac{1}{2} m \omega^{2} x^{2}
$$

assuming $x$ equal to $x_{o} \sin (\omega t)$. Also the speed is $u=\omega x_{o} \cos (\omega t)$. The aver- 
age value of the velocity-squared is $\overline{u^{2}}=\omega^{2} x_{o}^{2} / 2$. We will calculate the quantized circular frequency $\omega$ and quantized total energy $E$. Based on the relation (2.23) presented in subsection 2.4, the following relation occur:

$$
\overline{E_{k i n, n}}=\frac{1}{4} m \omega_{n}^{2} x_{o}^{2}=\frac{1}{2} h n v_{n}
$$

where $\omega_{n}=2 \pi v_{n}$, so with respect to quantized circular frequency, the resulting relation is:

$$
\omega_{n}=\frac{2 \hbar}{m x_{o}^{2}} n
$$

Therefore the total energy of the oscillator is given by:

$$
E_{n}=\frac{1}{2} m \omega_{n}^{2} x_{o}^{2}=n \hbar \omega_{n}
$$

The values $E_{k i n, n}$ of quantized kinetic energy, and the values $E_{d y n, n}$ of quantized dynamic energy, are:

$$
\begin{aligned}
& E_{k i n, n}=\frac{1}{2} m \omega_{n}^{2} x_{o}^{2} \cos ^{2}\left(\omega_{n} t\right)=n \hbar \omega_{n} \cos ^{2}\left(\omega_{n} t\right) \\
& E_{d y n, n}=\frac{1}{2} m \omega_{n}^{2} x_{o}^{2} \sin ^{2}\left(\omega_{n} t\right)=n \hbar \omega_{n} \sin ^{2}\left(\omega_{n} t\right)
\end{aligned}
$$

\subsection{Hydrogen Atom}

Our basic hypothesis here is that the electron trajectory is elliptical, and one focal point of the ellipse is the center of mass of the hydrogen atom (that is, lies in the nucleus). The orbital position of the electron in polar coordinates (reference [8], paragraph 3-7, THE KEPLER PROBLEM: INVERSE SQUARE LAW OF FORCE), is given by the relations:

$$
r=\frac{\beta}{1+\epsilon \cos \theta}, \quad \beta=a\left(1-\epsilon^{2}\right), \quad \epsilon=\frac{\sqrt{a^{2}-b^{2}}}{a}
$$

where $a$ and $b$ are the lengths of the semi-major and semi-minor axis of the ellipse respectively. The angular momentum is conserved, and is equal to:

$$
L=m \dot{\theta} r^{2}
$$

The force exerted to the electron is:

$$
F=-\frac{e^{2}}{r^{2}}=m \ddot{r}-m r \dot{\theta}^{2}=-\frac{L \dot{\theta}}{\beta}
$$

where $e$ is the charge of the electron. According to the last relation the angular momentum squared is:

$$
L^{2}=e^{2} \beta m
$$

The kinetic energy is:

$$
E_{k i n}=\frac{1}{2} m \dot{r}^{2}+\frac{L^{2}}{2 m r^{2}}
$$


According to the relations:

$$
\begin{aligned}
& \dot{r}^{2}=\frac{r^{4} \dot{\theta}^{2}}{\beta^{2}}(\epsilon \sin \theta)^{2} \\
& \frac{1}{r^{2}}=\frac{1}{\beta^{2}}(1+\epsilon \cos \theta)^{2}
\end{aligned}
$$

and relation (3.20), the relation for kinetic energy becomes:

$$
E_{k i n}=\frac{L^{2}}{2 m \beta^{2}}\left(1+\epsilon^{2}+2 \epsilon \cos \theta\right)
$$

The dynamic energy, according to relation (3.22), is:

$$
E_{d y n}=-\frac{e^{2}}{r}=-\frac{L^{2}}{m \beta^{2}}(1+\epsilon \cos \theta)
$$

The total energy of the electron as a sum of kinetic and dynamic energy (and according to the relation (3.22)) is:

$$
E=E_{k i n}+E_{d y n}=-\frac{L^{2}}{2 m \beta^{2}}\left(1-\epsilon^{2}\right)=-\frac{e^{2}}{2 a}
$$

From the last relation, it appears that the total energy of the electron is inversely proportional to the length of the large axis of the elliptical trajectory.

Then, in order to use the Equation (2.23) presented in subsection 2.4, we will calculate the time $T_{n}$ of a period. The area speed is constant and is expressed as:

$$
\frac{\mathrm{d} A}{\mathrm{~d} t}=\frac{1}{2} r^{2} \dot{\theta}=\frac{L}{2 m}
$$

The area of the ellipse, taking into account the area speed, is:

$$
\int_{0}^{T_{n}} \frac{\mathrm{d} A}{\mathrm{~d} t} \mathrm{~d} t=A=\frac{L T_{n}}{2 m}
$$

Since the area of ellipse is $A=\pi a b$, and based on relation (3.22), the calculated period (reference [8], paragraph 3-8, THE MOTION IN TIME IN THE KEPLER PROBLEM), is:

$$
T_{n}=\frac{2 m A}{L}=\frac{2 \pi \sqrt{m a^{3}}}{e}
$$

Since the frequency of periodic electron motion in the closed elliptical trajectory is $v_{n}=1 / T_{n}$, the second member of the Equation (2.23) presented in subsection 2.4 becomes:

$$
\overline{E_{k i n, n}}=\frac{1}{2} h n v_{n}=\frac{e \hbar}{2 \sqrt{m a^{3}}} n
$$

The time average of kinetic and dynamic energy (that is, the values of $\overline{E_{k i n}}$ and $\overline{E_{d y n}}$ ), are:

$$
\overline{E_{k i n}}=\frac{L^{2}}{2 m \beta^{2}}\left(1+\epsilon^{2}+2 \epsilon \overline{\cos \theta}\right)
$$




$$
\overline{E_{d y n}}=-\frac{L^{2}}{m \beta^{2}}(1+\epsilon \overline{\cos \theta})
$$

so, based on the virial theorem $2 \overline{E_{k i n}}+\overline{E_{d y n}}=0$ (reference [8], paragraph 3-4, THE VIRIAL THEOREM), the calculated time average of $\cos \theta$ is $\overline{\cos \theta}=-\epsilon$. Therefore, the average value of kinetic energy is:

$$
\overline{E_{k i n}}=\frac{L^{2}}{2 m \beta^{2}}\left(1-\epsilon^{2}\right)=\frac{e^{2}}{2 a}
$$

which is the expected value, since, due to the virial theorem, the total energy is $E=-\overline{E_{\text {kin }}}=-e^{2} /(2 a)$. Therefore, the Equation (2.23) presented in subsection 2.4 is expressed in the form:

$$
\overline{E_{k i n, n}}=\frac{e^{2}}{2 a_{n}}=\frac{e \hbar}{2 \sqrt{m a_{n}^{3}}} n
$$

so, the quantized semi-major axis of the elliptical trajectory is:

$$
a_{n}=\frac{\hbar^{2}}{m e^{2}} n^{2}
$$

The quantized total energy of the electron is:

$$
E_{n}=-\frac{e^{2}}{2 a_{n}}=-\frac{m e^{4}}{2 \hbar^{2}} \frac{1}{n^{2}}
$$

We will now examine the quantized term of kinetic energy $L^{2} /\left(2 m r^{2}\right)$, in order to determine the quantized angular momentum. Indeed, this term of kinetic energy obeys the relation (2.23) presented in subsection 2.4, i.e. the following equation:

$$
\overline{E_{k i n, \ell}}=\overline{\left(\frac{L^{2}}{2 m r^{2}}\right)}=\frac{1}{2} h \ell v_{\ell}
$$

where $\ell$ is integer quantum number, referring to the aforementioned term of kinetic energy. Since the angular momentum is $L=2 \pi v_{\ell} m r^{2}$, the time average of the quantity $1 / r^{2}$ is equal to $2 \pi v_{\ell} m / L$, so from the last relation the quantized angular momentum is:

$$
L=\ell \hbar
$$

Due to relation (3.22) and the relation (3.32), the resulting angular momentum is:

$$
L=\frac{b_{n, \ell}}{a_{n}} n \hbar
$$

where $b_{n, \ell}$ is the quantized semi-minor axis, and $n$ is the quantum number due to the quantized energy, while $\ell$ is the quantum number due to the quantized angular momentum.

From the last two equations we get the equation:

$$
\ell=\frac{b_{n, \ell}}{a_{n}} n
$$

If $b_{n, \ell}=a_{n}$ the trajectory is circular and the problem goes back to what was 
discussed earlier in this section. However, since we have assumed that the orbit is elliptical, it should be $b_{n, \ell}<a_{n}$. So, $\ell$ must be an integer smaller than $n$, that is $\ell=n-1, n-2, \cdots, 1,0$. The length of the semi-minor axis of the ellipse is calculated with the help of the last relation and the relation (3.32) as follows:

$$
b_{n, \ell}=\frac{\hbar^{2}}{m e^{2}} \ell n
$$

that is, it takes values:

$$
b_{n, \ell}=0,2 n \frac{\hbar^{2}}{m e^{2}}, 3 n \frac{\hbar^{2}}{m e^{2}}, \cdots,(n-1) n \frac{\hbar^{2}}{m e^{2}}
$$

We assume now that the elliptical orbit of the electron is on the level $X Y$ of a Cartesian coordinate system $X Y Z$. If the plane of the trajectory has been rotated at an angle $\varphi$, then the same angle is formed by the angular momentum vector with the $Z$ axis. The quantized projection of angular momentum on the $Z$ axis is given by the relation:

$$
L_{z}=\ell \hbar \cos \varphi=m_{\ell} \hbar
$$

where we have considered as an integer quantum number of the projection of angular momentum on the $Z$ axis the number $m_{\ell}$. It is called the magnetic quantum number because the application of an external magnetic field causes a splitting of spectral lines called the Zeeman effect ${ }^{5}$. From the last relation, since the quantity $\cos \varphi$ must take values in the range $-1 \leq \cos \varphi \leq 1$, the quantum number $m_{\ell}$ gets integer values in the range $-\ell \leq m_{\ell} \leq \ell$, that is, $m_{\ell}=0, \pm 1, \pm 2, \cdots, \pm \ell$.

\subsection{A simple Potential Step}

We assume the existence of an electric potential $V(x)$, constant in the $X$ direction, where $V(x)=0$ for $x<0$ and $V(x)=V_{0}$ for $x>0$. An electron beam is parallel to the $X$ axis, and the moving direction is from the negative to the positive semi-axis. The energy of the beam, in the area of the negative and the positive semi-axis remains constant, and the wave function will be in the form of a flat electromagnetic wave, which comes from the particle waves. The wave function on the negative semi-axis, can be represented as $\Psi_{i}=A_{i} \mathrm{e}^{i k x}$ for the incident beam and as $\Psi_{r}=A_{r} \mathrm{e}^{-i k x}$ for the reflected wave, where $A_{i}$ and $A_{r}$ are the corresponding complex amplitudes, while on the positive semi-axis the refracted wave function is represented as $\Psi_{t}=A_{t} \mathrm{e}^{\mathrm{i} k^{\prime} x}$, where $A_{t}$ is the complex amplitude.

We also assume that the kinetic energy of an electron of the incident-beam is $E$. Due to the energy conservation, the total energy of an electron of the refracted beam is $E=E_{k i n}^{\prime}+V_{0}$. The corresponding momentums are $p=\hbar k$ and

${ }^{5}$ When an external magnetic field is applied, sharp spectral lines like the $n=3 \rightarrow 2$ transition of hydrogen split into multiple closely spaced lines. First observed by Pieter Zeeman, this splitting is attributed to the interaction between the magnetic field and the magnetic dipole moment associated with the orbital angular momentum. In the absence of the magnetic field, the hydrogen energies depend only upon the principal quantum number $n$, and the emissions occur at a single wavelength. 
$p^{\prime}=\hbar k^{\prime}$, where $k=2 \pi / \lambda_{q}$ is the wave number of an electron of the incident or reflected beam, and $p^{\prime}=\hbar k^{\prime}$ is the wave number of an electron of the refracted beam. According to the relation (2.5) presented in subsection 2.1 and equation $E=E_{\text {kin }}=p^{2} /(2 m)$ the wave number $k$ is:

$$
k=\frac{\sqrt{2 m E}}{\hbar}
$$

while the wave number $k^{\prime}$ is:

$$
k^{\prime}=\frac{\sqrt{2 m E_{k i n}^{\prime}}}{\hbar}=\frac{\sqrt{2 m\left(E-V_{0}\right)}}{\hbar}
$$

We initially consider $E>V_{0}$. We designate as a refractive index, in the region of the potential $V_{0}$, the quantity $\eta=\lambda_{q} / \lambda_{q}^{\prime}=k^{\prime} / k$. In the area of the negative semi-axis, where the potential is zero, the refractive index is considered to be equal to the unit. The reflection and transmission are derived from the Fresnel types (reference [7], paragraph 1.5.2, Fresnel formulae):

$$
\begin{gathered}
\mathcal{R}=\left(\frac{\eta-1}{\eta+1}\right)^{2}=\left(\frac{k-k^{\prime}}{k+k^{\prime}}\right)^{2} \\
\mathcal{T}=\frac{4 \eta}{(\eta+1)^{2}}=\frac{4 k k^{\prime}}{\left(k+k^{\prime}\right)^{2}}
\end{gathered}
$$

In the case where $E<V_{0}, k^{\prime}$ and refractive index $\eta$ are imaginary numbers, the reflection is equal to the unit, while the transmission is equal to zero (total reflection).

Another way to deal with the same problem is to calculate the reflection and refraction from the expressions for the particle currents ${ }^{6}$. Since the above wave functions refer to the incident, reflected, and refracted beam, the quantity $|\Psi|^{2}$ express the particle density (i.e., the number of particles in the volume unit). For example, for $\gamma \simeq 1$, the quantity $\left|\Psi_{i}\right|^{2} u=\left|A_{i}\right|^{2} \hbar k / m$ is proportional to the current of the incident beam. Also, the quantities $\left|\Psi_{r}\right|^{2} u=\left|A_{r}\right|^{2} \hbar k / m$ and $\left|\Psi_{t}\right|^{2} u=\left|A_{t}\right|^{2} \hbar k^{\prime} / m$ are proportional to the reflected and refracted currents respectively. The reflection and transmission are given by the following relations:

$$
\begin{gathered}
\mathcal{R}=\frac{J_{r}}{J_{i}}=\frac{\left|A_{r}\right|^{2} \frac{\hbar k}{m}}{\left|A_{i}\right|^{2} \frac{\hbar k}{m}}=\frac{\left|A_{r}\right|^{2}}{\left|A_{i}\right|^{2}} \\
\mathcal{T}=\frac{J_{t}}{J_{i}}=\frac{\left|A_{t}\right|^{2} \frac{\hbar k^{\prime}}{m}}{\left|A_{i}\right|^{2} \frac{\hbar k}{m}}=\frac{\left|A_{t}\right|^{2}}{\left|A_{i}\right|^{2}} \frac{k^{\prime}}{k}
\end{gathered}
$$

where, the $J_{i}, J_{r}, J_{t}$ are the currents of the reflected and refracted beam respectively.

In the area of the negative semi-axis the total wave function (incident and reflected beam) is given by the relation: 


$$
\Psi_{-}(x)=\Psi_{i}(x)+\Psi_{r}(x)
$$

while in the area of the positive semi-axis (refracted beam only):

$$
\Psi_{+}(x)=\Psi_{t}(x)
$$

The continuity boundary conditions at $x=0$ impose equality $\Psi_{-}(0)=\Psi_{+}(0)$ and also the equality of the first derivatives at the same point $\Psi_{-}^{\prime}(0)=\Psi_{+}^{\prime}(0)$. Due to these conditions, the following relations arise:

$$
\begin{gathered}
A_{i}+A_{r}=A_{t} \\
i k A_{i}+i k A_{r}=i k^{\prime} A_{t}
\end{gathered}
$$

from which relations emerge:

$$
\begin{aligned}
& \frac{A_{r}}{A_{i}}=\frac{k-k^{\prime}}{k+k^{\prime}} \\
& \frac{A_{t}}{A_{i}}=\frac{2 k}{k+k^{\prime}}
\end{aligned}
$$

From the last two relations and from relations (3.44) and (3.45), we end up with the previous relations for reflection and transmission.

\subsection{A Rectangular Potential Step}

we consider in this example a fixed potential in the $X$ direction in the region $0<x<L$ equal to $V_{0}$, while everywhere else (for $x<0$ and for $x>L$ ) the potential is zero. We denote with $a$ the region where $x<0, b$ is the region where $0<x<L$ and with $c$ the region where $x>L$. We also define as $\Psi_{a, i}=A_{a, i} \mathrm{e}^{i k x}$ and $\Psi_{a, r}=A_{a, r} \mathrm{e}^{-i k x}$ the wave functions of the incident and reflected beam, respectively, in the region $a$. In the region $b$ we define as $\Psi_{b, t}=A_{b, t} \mathrm{e}^{i k^{\prime} x}$ and $\Psi_{b, r}=A_{b, r} \mathrm{e}^{-i k^{\prime} x}$ the wave function of the refracted and reflected beam, respectively, while in the region $c$ we define as the $\Psi_{c, t}=A_{c, t} e^{i k x}$ the wave function of the unique beam that is transmitted in this area. The other components of the beam, created by new reflections and refractions (second order or higher), are considered negligible. The refractive index, as in the previous example, has a value equal to the unit in the region where the potential is zero, while at region $b$, where the potential is $V_{0}$, has a value of $\eta=\lambda_{q} / \lambda_{q}^{\prime}=k^{\prime} / k$.

In the case where the kinetic energy $E$ of an electron of the incident beam is greater than the dynamic energy $V_{0}$ of the region $b$, according to the previous example, the refractive index $\eta$ will have a real value. Also based on the conservation principle, the energy of any electron in the $b$ region will be equal to $E=E_{k i n}^{\prime}+V_{0}$, while in the $a$ and $c$ regions it will have only kinetic energy equal to $E$. This case is equivalent to that of an electromagnetic wave incident perpendicular to a dielectric plate of a width $L$, so the reflection will be (reference [7], paragraph 1.6.4, A homogeneous dielectric film):

${ }^{6}$ The same way of dealing with this example, based on Schrodinger's quantum mechanics, is set out in a lecture-note entitled "Reflection and Transmission at a Potential Step", MIT OpenCourseWare, http://ocw.mit.edu, 6.007 Electromagnetic Energy: From Motors to Lasers, Spring 2011. 


$$
\mathcal{R}=\frac{r_{a b}^{2}+r_{b c}^{2}+2 r_{a b} r_{b c} \cos \left(2 k^{\prime} L\right)}{1+r_{a b}^{2} r_{b c}^{2}+2 r_{a b} r_{b c} \cos \left(2 k^{\prime} L\right)}
$$

where $r_{a b}=(1-\eta) /(1+\eta)$ and $r_{b c}=(\eta-1) /(\eta+1)$. From this equation, according to the relation $\eta=k^{\prime} / k$, we get:

$$
\mathcal{R}=\frac{\left(k^{2}-k^{\prime 2}\right)^{2} \sin ^{2}\left(k^{\prime} L\right)}{4 k^{2} k^{\prime 2}+\left(k^{2}-k^{\prime 2}\right)^{2} \sin ^{2}\left(k^{\prime} L\right)}
$$

Also, following the analogous procedure for the transmission, we get the relation:

$$
\mathcal{T}=\frac{4 k^{2} k^{\prime 2}}{4 k^{2} k^{\prime 2}+\left(k^{2}-k^{\prime 2}\right)^{2} \sin ^{2}\left(k^{\prime} L\right)}
$$

We will now follow the methodology on particle currents and boundary conditions of continuity, as in the previous example. In this case the reflection and transmission are derived from the corresponding current ratios, according to the following relations:

$$
\begin{gathered}
\mathcal{R}=\frac{J_{a, r}}{J_{a, i}}=\frac{\left|A_{a, r}\right|^{2} \frac{\hbar k}{m}}{\left|A_{a, i}\right|^{2} \frac{\hbar k}{m}}=\frac{\left|A_{a, r}\right|^{2}}{\left|A_{a, i}\right|^{2}} \\
\mathcal{T}=\frac{J_{c, t}}{J_{a, i}}=\frac{\left|A_{c, t}\right|^{2} \frac{\hbar k}{m}}{\left|A_{a, i}\right|^{2} \frac{\hbar k}{m}}=\frac{\left|A_{c, t}\right|^{2}}{\left|A_{a, i}\right|^{2}}
\end{gathered}
$$

The total wave function in region $a$ is defined as $\Psi_{a}(x)=\Psi_{a, i}(x)+\Psi_{a, r}(x)$, in region $b$ as $\Psi_{b}(x)=\Psi_{b, t}(x)+\Psi_{b, r}(x)$, and in region $c$ as $\quad \Psi_{c}(x)=\Psi_{c, t}(x)$. The boundary conditions of continuity, for the total wave functions and the first derivatives at $x=0$ and $x=L$, are:

$$
\begin{aligned}
& \Psi_{a}(0)=\Psi_{b}(0) \\
& \Psi_{a}^{\prime}(0)=\Psi_{b}^{\prime}(0) \\
& \Psi_{b}(L)=\Psi_{c}(L) \\
& \Psi_{b}^{\prime}(L)=\Psi_{c}^{\prime}(L)
\end{aligned}
$$

From these equations, four relations between the complex amplitudes are taken, which are the following:

$$
\begin{aligned}
& A_{a, i}+A_{a, r}=A_{b, t}+A_{b, r} \\
& i k\left(A_{a, i}-A_{a, r}\right)=i k^{\prime}\left(A_{b, t}-A_{b, r}\right) \\
& A_{b, t} \mathrm{e}^{i k^{\prime} L}+A_{b, r} \mathrm{e}^{-i k^{\prime} L}=A_{c, t} \mathrm{e}^{i k L} \\
& i k^{\prime}\left(A_{b, t} \mathrm{e}^{i k^{\prime} L}-A_{b, r} \mathrm{e}^{-i k^{\prime} L}\right)=i k A_{c, t} \mathrm{e}^{i k L}
\end{aligned}
$$

From the last four equations we get the equality:

$$
\frac{A_{a, r}}{A_{a, i}}=\frac{i\left(k^{\prime 2}-k^{2}\right) \sin \left(k^{\prime} L\right)}{2 k k^{\prime} \cos \left(k^{\prime} L\right)-i\left(k^{\prime 2}+k^{2}\right) \sin \left(k^{\prime} L\right)}
$$


From this last equality and from the relations (3.55) and (3.56), we reach the same relation for the reflection, that is the relation (3.53). In the same way, the transmission, given by the relation (3.54), is also calculated.

In the case where the kinetic energy $E$ of an electron of the incident beam is less than the dynamic energy $V_{0}$ of the region $b$, the refractive index $\eta$ and the wavenumber $k^{\prime}$ are imaginary quantities, and the $k^{\prime}$ should be replaced with $i k^{\prime}$, which is equal to $i \sqrt{2 m\left(V_{0}-E\right)} / \hbar$. Therefore the reflection and transmission are:

$$
\begin{aligned}
& \mathcal{R}=\frac{\left(k^{2}+k^{\prime 2}\right)^{2} \sinh ^{2}\left(k^{\prime} L\right)}{4 k^{2} k^{\prime 2}+\left(k^{2}+k^{\prime 2}\right)^{2} \sinh ^{2}\left(k^{\prime} L\right)} \\
& \mathcal{T}=\frac{4 k^{2} k^{\prime 2}}{4 k^{2} k^{\prime 2}+\left(k^{2}+k^{\prime 2}\right)^{2} \sinh ^{2}\left(k^{\prime} L\right)}
\end{aligned}
$$

Replacing the $k$ and $k^{\prime}$, for $E>V_{0}$, according to the relations $k=\sqrt{2 m E} / \hbar$ and $k^{\prime}=\sqrt{2 m\left(E-V_{0}\right)} / \hbar$, reflection and transmission become:

$$
\begin{aligned}
& \mathcal{R}=\frac{V_{0}^{2} \sin ^{2}\left(L \sqrt{2 m\left(E-V_{0}\right)} / \hbar\right)}{4 E\left(E-V_{0}\right)+V_{0}^{2} \sin ^{2}\left(L \sqrt{2 m\left(E-V_{0}\right)} / \hbar\right)} \\
& \mathcal{T}=\frac{4 E\left(E-V_{0}\right)}{4 E\left(E-V_{0}\right)+V_{0}^{2} \sin ^{2}\left(L \sqrt{2 m\left(E-V_{0}\right)} / \hbar\right)}
\end{aligned}
$$

while for $E<V_{0}$, according to the equations $k=\sqrt{2 m E} / \hbar$ and $k^{\prime}=\sqrt{2 m\left(V_{0}-E\right)} / \hbar$, the reflection and transmission are:

$$
\begin{aligned}
& \mathcal{R}=\frac{V_{0}^{2} \sinh ^{2}\left(L \sqrt{2 m\left(V_{0}-E\right)} / \hbar\right)}{4 E\left(V_{0}-E\right)+V_{0}^{2} \sinh ^{2}\left(L \sqrt{2 m\left(V_{0}-E\right)} / \hbar\right)} \\
& \mathcal{T}=\frac{4 E\left(V_{0}-E\right)}{4 E\left(V_{0}-E\right)+V_{0}^{2} \sinh ^{2}\left(L \sqrt{2 m\left(V_{0}-E\right)} / \hbar\right)}
\end{aligned}
$$

\section{Quantum Electrodynamics}

We will examine in this section quantum electrodynamics, according to classical theory based on the absolute reference system. Can a theory of classical physics to include quantum field theory and give corresponding theoretical results?

The answer is affirmative, and this will be seen in this study, in this section. The waveforms used in this section, as in the previous ones, are solutions of this wave equation and do not express probability amplitudes, as they derive from a probabilistic view. In contrast, that waveforms express the propagation of a peculiar particle electromagnetic wave, according to the assumption of absolute reference system, which is a classic non-relativistic view of nature in the broader field of statistical physics.

The free-particle wave-equation for high velocities, according to the hypothe- 
sis of the absolute reference system, is the wave-equation, in which the force currier is massive and the factor $1 / c^{2}$ has been replaced by $1 /\left(\gamma^{2} c^{2}\right)$, since velocity and momentum are the Newtonian velocity and momentum multiplied by the Lorentz coefficient $\gamma$, while the term $m^{2} c^{2} / \hbar^{2}$ is a constant term, which is independent of speed and is due to internal particle energy. This wave-equation is given by the relation:

$$
\frac{1}{\gamma^{2} c^{2}} \frac{\partial^{2} \Psi}{\partial t^{2}}=\nabla^{2} \Psi-\frac{m^{2} c^{2}}{\hbar^{2}} \Psi
$$

This equation looks like the Klein-Gordon equation ${ }^{7}$. The wave function that is a solution of this equation will be that of the plane wave ${ }^{8}$, which is in the form of:

$$
\Psi(\boldsymbol{r}, t)=N \mathrm{e}^{i(\boldsymbol{k} \cdot \boldsymbol{r}-\omega t)}
$$

where $N$ is the normalization factor, which will be discussed below. By substituting this wave function in the previous differential equation we get the following relation:

$$
\frac{\omega^{2}}{\gamma^{2} c^{2}}=\boldsymbol{k}^{2}+\frac{m^{2} c^{2}}{\hbar^{2}}=\frac{1}{\hbar^{2}}\left(\boldsymbol{p}^{2}+m^{2} c^{2}\right)
$$

so, due to the energy relation $E=\boldsymbol{p}^{2} /(2 m)+(1 / 2) m c^{2}$, we obtain the following equation:

$$
\omega=\frac{2 E}{\hbar}
$$

According to this last relation the wave function (4.2) takes the form:

$$
\Psi(\boldsymbol{r}, t)=N \mathrm{e}^{i(p \cdot r-2 E t) / \hbar}
$$

The operator which acts on the wave function $\Psi(\boldsymbol{r}, t)$ and gives as an eigen value the particle's momentum $\boldsymbol{p}$ is

$$
\hat{\boldsymbol{p}}=-i \hbar \nabla
$$

while the differential operator, which yields as an eigenvalue the energy $E$ is

$$
\hat{E}=\frac{i \hbar}{2} \frac{\partial}{\partial t}
$$

Multiplying the two members of the Equation (4.1) by the quantity $i \Psi^{*}$, the following equation and its conjugate arises:

$$
\begin{gathered}
\frac{i}{\gamma^{2} c^{2}} \Psi^{*} \frac{\partial^{2} \Psi}{\partial t^{2}}=i \Psi^{*} \nabla^{2} \Psi-i \frac{m^{2} c^{2}}{\hbar^{2}} \Psi^{*} \Psi \\
-\frac{i}{\gamma^{2} c^{2}} \Psi \frac{\partial^{2} \Psi^{*}}{\partial t^{2}}=-i \Psi \nabla^{2} \Psi^{*}+i \frac{m^{2} c^{2}}{\hbar^{2}} \Psi \Psi^{*}
\end{gathered}
$$

Adding the last two equations and multiplying with $\hbar /(2 m)$, so that dimen${ }^{7}$ ref. [9], Chapter 2, Single-particle relativistic wave equations, section 2.2, Klein-Gordon equation.

${ }^{8}$ ref. [10], CHAPTER 6, paragraph 48, Monochromatic plane waves. 
sions to do consistent with the Equation (2.12) presented in subsection 2.2, we obtain the following equation of continuity:

$$
\frac{\partial}{\partial t}\left(\frac{i \hbar}{2 m \gamma^{2} c^{2}}\left(\Psi^{*} \frac{\partial \Psi}{\partial t}-\Psi \frac{\partial \Psi^{*}}{\partial t}\right)\right)=-\nabla \cdot\left(\frac{i \hbar}{2 m}\left(\Psi \nabla \Psi^{*}-\Psi^{*} \nabla \Psi\right)\right)
$$

The particle density and the particle current are given by the relations:

$$
\begin{gathered}
\rho=\frac{i \hbar}{2 m \gamma^{2} c^{2}}\left(\Psi^{*} \frac{\partial \Psi}{\partial t}-\Psi \frac{\partial \Psi^{*}}{\partial t}\right) \\
\boldsymbol{J}=\frac{i \hbar}{2 m}\left(\Psi \nabla \Psi^{*}-\Psi^{*} \nabla \Psi\right)
\end{gathered}
$$

According to relations $\hat{E} \Psi=\frac{i \hbar}{2} \frac{\partial \Psi}{\partial t}=\frac{1}{2} m \gamma^{2} c^{2} \Psi$ and $\hat{p} \Psi=-i \hbar \nabla \Psi=\boldsymbol{p} \Psi$, the last two relations give:

$$
\begin{gathered}
\rho=\Psi \Psi^{*} \\
\boldsymbol{J}=\frac{\boldsymbol{p}}{m} \Psi \Psi^{*}=\rho \gamma \boldsymbol{u}
\end{gathered}
$$

because the speed $\boldsymbol{u}$ is measured by the clock of laboratory inertial system, while when it is measured by the clock of reference system of the particle (or particle parallel beam) the speed is equal to $\gamma \boldsymbol{u}$.

\subsection{Yukawa Potential}

Now we will extend to the study of the interaction between heavier particles and the corresponding dynamics. This study has to be done from the point of view of the hypothesis of the absolute system of reference. Such a potential we will first examine is that which comes from relatively heavier particles, such as protons and neutrons. In particular, we will calculate the potential comes from the exchange of intermediate particles (force carriers) that give rise to forces between such heavy particles (see [2], paragraph 1.5.2, The Yukawa Theory).

The set of carrier particles in the field around a proton, in addition to the photons that are carriers of electrostatic interactions, consists of larger photon packets, which are the intermediate particles of interactions between nucleons. These particles, which are the carriers of strong interactions, are the explanation of the small radius of force action between the nucleons inside the atomic nucleus. The radius of action of static interactions depends, on the mass of the carrier of the quantum field, and an explanation for this was given by Yukawa in 1935 , in his effort to describe the above-mentioned forces.

We assume initially that the mass of an intermediate particle that is exchanged is $m$. From a physical point of view, this exchange gives momentum that justifies the existing force of interaction. Along with the capturing of this mass, the heavy body takes extra energy equal to the total kinetic energy of this intermediate particle. This energy is:

$$
E=\frac{1}{2} m \gamma^{2} c^{2}=\frac{p^{2}}{2 m}+\frac{1}{2} m c^{2}
$$


where $p=m \gamma u$ is the momentum, $\gamma=\left(1-u^{2} / c^{2}\right)^{-1 / 2}$ and $u$ is the velocity. Under these conditions, the following relation, between momentum squared and energy squared, is taken:

$$
p^{2}+m^{2} c^{2}=\frac{1}{\gamma^{2} c^{2}}(2 E)^{2}
$$

The differential equation of wave motion results from the replacement, in the last relation, of $p$ and $E$ with the corresponding operators $\hat{p}=-i \hbar \nabla$ and $\hat{E}=(i \hbar / 2) \partial / \partial t$, and is:

$$
\nabla^{2} \Psi-\frac{m^{2} c^{2}}{\hbar^{2}} \Psi-\frac{1}{\gamma^{2} c^{2}} \frac{\partial^{2} \Psi}{\partial t^{2}}=0
$$

This last equation has two partial solutions. One partial solution is given by the Equation (4.4), but, at the present, we are not interested as much in the propagation of the particle-wave, as much we are interested in the examined here static potential. The other solution is of the form:

$$
U(r)=\frac{g}{4 \pi r} \mathrm{e}^{-m c r / \hbar}
$$

and is independent of time. The $U(r)$ function is the known Yukawa potential. The quantity $g$ is a constant, resulting from this solution of differential wave-equation, and is determined by the intensity of the point source.

The physical analog of Yukawa potential in electromagnetism is that resulting from the substitution of the constant $g$ with the charge $q$. However, because of the very small mass of the interaction photon, the exponential part of the potential is very close to 1 and therefore the electrical potential is of the known form $U_{e l}(r)=q /(4 \pi r)$. The $g$ constant in Yukawa theory plays the same role as the charge in electrostatics and measures the "strong nuclear charge".

According to the history of nuclear forces the Yukawa hypothesis predicted as carrier of strong interactions a spinless quantum of mass

$m c^{2}=\hbar c / R \simeq 100 \mathrm{MeV}$. The pion observed in 1947 had mass $\simeq 140 \mathrm{MeV}$, spin 0 , and was assigned as the nuclear-force quantum.

\subsection{Perturbation Theory}

Let us assume that a time depended potential $V(r, t)$ causes a correspondingly change in kinetic energy, at the time $t$, equal to $\Delta T$, that is $V(\boldsymbol{r}, t)=\Delta T$. We introduce the concept of a "probable wave-function", by which the evolution of the perturbation is described, assuming that the expansion of that wave-function is a series:

$$
\Psi_{p}(\boldsymbol{r}, t)=\sum_{n=0}^{\infty} a_{n}(t) \Psi_{n}(\boldsymbol{r}, t)=\sum_{n=0}^{\infty} a_{n}(t) \Phi_{n}(\boldsymbol{r}) \mathrm{e}^{-i 2 E_{n} t / \hbar}=\Delta T
$$

where $a_{n}(t)$ complex time dependent coefficients, $E_{n}=(1 / 2) m \gamma_{n}^{2} c^{2}$, $\gamma_{n}=\left(1-u_{n}^{2} / c^{2}\right)^{-1 / 2}, \quad \Psi_{m}(\boldsymbol{r}, t)=\Phi_{m}(\boldsymbol{r}) \mathrm{e}^{-i 2 E_{m} t / \hbar}$ solutions of the wave Equation (4.1), and $\Phi_{m}(\boldsymbol{r})$ spatial wave functions that obey the equation $\frac{\hat{p}^{2}}{2 m} \Phi_{m}=-\frac{\hbar^{2}}{2 m} \nabla^{2} \Phi_{m}=\frac{\boldsymbol{p}^{2}}{2 m} \Phi_{m}$ and form an orthonormal basis, according to 
the relation:

$$
\int \Phi_{m}^{*}(\boldsymbol{r}) \Phi_{n}(\boldsymbol{r}) \mathrm{d}^{3} \boldsymbol{r}=\delta_{m n}
$$

and $\delta_{m n}$ the Kronecker delta.

Also the "probable wave-function" $\Psi_{p}(\boldsymbol{r}, t)$ is normalized so that:

$$
\int_{-\infty}^{+\infty} \mathrm{d} t \int \mathrm{d}^{3} \boldsymbol{r} \Psi_{p}(\boldsymbol{r}, t) \Psi_{p}^{*}(\boldsymbol{r}, t)=1
$$

so $\sum_{n=0}^{\infty}\left|a_{n}(t)\right|^{2}=1$. Since the wave function $\Psi_{p}(\boldsymbol{r}, t)$, as we have defined it, is a probable wave function, a time dependent factor $a_{n}(t)$ expresses the probability amplitude of transition from excited state to state $n$, at moment $t$. This probability is equal to $\left|a_{n}(t)\right|^{2}=a_{n}(t) a_{n}^{*}(t)$, where $n=0,1,2,3, \cdots$.

Assuming that before action of the potential $V(\boldsymbol{r}, t)$ the kinetic energy is $T_{i}=p^{2} /(2 m)$, then during the perturbation at time $t$, the total transport kinetic energy will be equal to $T=T_{i}+\Delta T=T_{i}+V(\boldsymbol{r}, t)$ and the corresponding operator is $\hat{T}=\hat{T}_{i}+V=\hat{p}^{2} /(2 m)+V(\boldsymbol{r}, t)$, where the total kinetic energy (that is the sum of internal and transport kinetic energy) is equal to $E=T_{i}+\Delta T+(1 / 2) m c^{2}=T_{i}+V(\boldsymbol{r}, t)+(1 / 2) m c^{2}$. If we denote by $E_{o}$ the total kinetic energy of the initial state, that is before action of the potential $V(\boldsymbol{r}, t)$, then $E_{o}=T_{i}+(1 / 2) m c^{2}$ and the corresponding operator is $\hat{E}_{o}=\hat{p}^{2} /(2 m)+(1 / 2) m c^{2}$. The operator of total kinetic energy is

$$
\hat{E}=\frac{i \hbar}{2} \frac{\partial}{\partial t}=\hat{E}_{o}+V(\boldsymbol{r}, t)
$$

so we get the following differential equation:

$$
\frac{i \hbar}{2} \frac{\partial \Psi_{p}}{\partial t}=\left(\hat{E}_{o}+V(\boldsymbol{r}, t)\right) \Psi_{p}
$$

By substituting the expression 15 in this last differential equation, since $E_{n}=p_{n}^{2} /(2 m)+(1 / 2) m c^{2}$, is taken the following equation:

$$
\frac{i \hbar}{2} \sum_{n=0}^{\infty} \frac{\mathrm{d} a_{n}(t)}{\mathrm{d} t} \Phi_{n}(\boldsymbol{r}) e^{-i 2 E_{n} t / \hbar}=\sum_{n=0}^{\infty} V(\boldsymbol{r}, t) a_{n}(t) \Phi_{n}(\boldsymbol{r}) \mathrm{e}^{-i 2 E_{n} t / \hbar}
$$

By multiplication of the last equation from the left with $\Psi_{f}^{*}=\Phi_{f}^{*}(\boldsymbol{r}) \mathrm{e}^{-i 2 E_{n} t / \hbar}$ and by integration over $\boldsymbol{r}$, is obtained

$$
\begin{aligned}
& \frac{i \hbar}{2} \sum_{n=0}^{\infty} \frac{\mathrm{d} a_{n}(t)}{\mathrm{d} t} \int \mathrm{d}^{3} \boldsymbol{r} \Phi_{f}^{*}(\boldsymbol{r}) \Phi_{n}(\boldsymbol{r}) \mathrm{e}^{-i 2\left(E_{n}-E_{f}\right) t / \hbar} \\
& =\sum_{n=0}^{\infty} a_{n}(t) \int \mathrm{d}^{3} \boldsymbol{r} \Phi_{f}^{*}(\boldsymbol{r}) V(\boldsymbol{r}, t) \Phi_{n}(\boldsymbol{r}) \mathrm{e}^{-i 2\left(E_{n}-E_{f}\right) t / \hbar}
\end{aligned}
$$

Using the orthonormality relation for $\Phi_{m}$ we then arrive at the following coupled linear differential equation for $a_{n}(t)$,

$$
\frac{i \hbar}{2} \frac{\mathrm{d} a_{k}(t)}{\mathrm{d} t}=\sum_{n=0}^{\infty} a_{n}(t) V_{k n} \mathrm{e}^{i \omega_{k n} t / \hbar}
$$

where we have defined

$$
\omega_{k n}=\omega_{k}-\omega_{n}=2\left(E_{k}-E_{n}\right) / \hbar
$$


and what is sometimes called the transition matrix element:

$$
V_{k n}(t)=\int \mathrm{d}^{3} \boldsymbol{r} \Phi_{k}^{*}(\boldsymbol{r}) V(\boldsymbol{r}, t) \Phi_{n}(\boldsymbol{r})
$$

According to the first order approximation, the Equation (4.22) gives us

$$
\frac{i \hbar}{2} \frac{\mathrm{d} a_{k}(t)}{\mathrm{d} t}=V_{k i}(t) \mathrm{e}^{2 i\left(E_{k}-E_{i}\right) t / \hbar}
$$

Using that $\lim _{t \rightarrow-\infty} a_{k}(t)=0$ and integrating this equation we obtain for the coefficient $a_{k}(t)$ at time $t$,

$$
a_{k}(t)=\int_{-\infty}^{t} \frac{\mathrm{d} a_{k}\left(t^{\prime}\right)}{\mathrm{d} t^{\prime}} \mathrm{d} t^{\prime}=\frac{2}{i \hbar} \int_{-\infty}^{t} V_{k i}\left(t^{\prime}\right) \mathrm{e}^{2 i\left(E_{k}-E_{i}\right) t^{\prime} / \hbar} \mathrm{d} t^{\prime}
$$

for $k \neq i$. We define the transition amplitude $\mathcal{A}_{f i}$ as the amplitude to go from a state $i$ to a final state $f$ at large times,

$$
\mathcal{A}_{f i}=\lim _{t \rightarrow \infty} a_{k}(t)=\frac{2}{i \hbar} \int_{-\infty}^{+\infty} \mathrm{d} t \int \mathrm{d}^{3} \boldsymbol{r} \Psi_{f}^{*}(\boldsymbol{r}, t) V(\boldsymbol{r}, t) \Psi_{i}(\boldsymbol{r}, t)
$$

For a potential that is time-independent the expression, for the transition amplitude, becomes

$$
\mathcal{A}_{f i}=\frac{2 V_{f i}}{i \hbar} \int_{-\infty}^{+\infty} \mathrm{e}^{i \omega_{f i} t / \hbar} \mathrm{d} t=-2 \pi i V_{f i} \delta\left(E_{f}-E_{i}\right)
$$

where

$$
\delta\left(E_{f}-E_{i}\right)=\frac{2}{\hbar} \lim _{T \rightarrow \infty}\left(\frac{1}{2 \pi} \int_{-T / 2}^{+T / 2} \mathrm{e}^{2 i\left(E_{f}-E_{i}\right) t / \hbar} \mathrm{d} t\right)
$$

We define the mean transition rate in the limit for large $T$ as

$$
W_{f i}=\lim _{T \rightarrow \infty} \frac{\left|\mathcal{A}_{f i}\right|^{2}}{T}
$$

When the wave functions are those of the plane wave, that is $\Psi(\boldsymbol{r}, t)=N \mathrm{e}^{-i(2 E t-\boldsymbol{p} \cdot \boldsymbol{r}) / \hbar}$, we apply so-called box normalization, that is, we choose a finite volume $V$ and normalize all wave functions such that

$$
\int_{V} \Psi^{*}(\boldsymbol{r}, t) \Psi(\boldsymbol{r}, t) \mathrm{d}^{3} \boldsymbol{r}=1
$$

For the plane waves this gives $N=1 / \sqrt{V}$. For an incident particle, the flux factor $F$ is defined as:

$$
F=\left|J_{i}\right|=\frac{1}{V} \gamma\left|\boldsymbol{u}_{i}\right|
$$

where, for plane waves $1 / V=\rho=\Psi \Psi^{*}=N N^{*}=|N|^{2}, \quad \boldsymbol{u}_{i}$ is the velocity of the particle, and according to the previous $\boldsymbol{J}_{i}=\rho \gamma \boldsymbol{u}_{i}$.

The phase space factor for a process with $n$ final state particles is

$$
\mathrm{d} \Phi=\prod_{f=1}^{n} \frac{V}{(2 \pi)^{3}} \mathrm{~d}^{3} \boldsymbol{p}_{f}
$$

The cross-section is 


$$
\mathrm{d} \sigma=\frac{1}{F} W_{f i} \mathrm{~d} \Phi
$$

\subsection{Coulomb Scattering}

We will examine the case where the outer field comes from a point charge at the beginning of the axes, while the velocity of the incoming charged particle is low, that is $\gamma \simeq 1$. In this case the expression for the electrical potential, in the Gaussian unit system, is

$$
V(r)=\frac{Z e}{4 \pi|r|}
$$

The wave function of the initial state and the conjugate wave function of the final state are:

$$
\begin{gathered}
\Psi_{i}=N_{i} \mathrm{e}^{i\left(\boldsymbol{p}_{i} \cdot \boldsymbol{r}-2 E_{i} t\right) / \hbar} \\
\Psi_{f}^{*}=N_{f}^{*} \mathrm{e}^{-i\left(\boldsymbol{p}_{f} \cdot \boldsymbol{r}-2 E_{f} t\right) / \hbar}
\end{gathered}
$$

The transition amplitude is:

$$
\mathcal{A}_{f i}=\frac{2}{i \hbar} \int_{-\infty}^{+\infty} \mathrm{d} t \int \mathrm{d}^{3} \boldsymbol{r} N_{i} N_{f}^{*} \mathrm{e}^{i\left(\boldsymbol{p}_{i}-\boldsymbol{p}_{f}\right) \cdot \boldsymbol{r} / \hbar} \mathrm{e}^{i\left(2 E_{f}-2 E_{i}\right) t / \hbar} \frac{Z e^{2}}{4 \pi|\boldsymbol{r}|}
$$

according to the relations:

$$
\begin{gathered}
\frac{1}{|\boldsymbol{q}|^{2}}=\int \mathrm{d}^{3} \boldsymbol{r} \mathrm{e}^{i \boldsymbol{q} \cdot \boldsymbol{r}} \frac{1}{4 \pi|\boldsymbol{r}|} \\
\int_{-\infty}^{+\infty} \mathrm{e}^{i\left(2 E_{f}-2 E_{i}\right) t / \hbar} \mathrm{d} t=\frac{\hbar}{2} 2 \pi \delta\left(E_{f}-E_{i}\right)
\end{gathered}
$$

By setting $\boldsymbol{q}=\boldsymbol{p}_{i} / \hbar-\boldsymbol{p}_{f} / \hbar$, the transition amplitude will be:

$$
\mathcal{A}_{f i}=-i N_{i} N_{f}^{*} 2 \pi \delta\left(E_{f}-E_{i}\right) \frac{Z e^{2} \hbar^{2}}{\left|\boldsymbol{p}_{i}-\boldsymbol{p}_{f}\right|^{2}}
$$

The time-averaged transition rate is:

$$
W_{f i}=\lim _{T \rightarrow \infty} \frac{\left|\mathcal{A}_{f i}\right|^{2}}{T}=\left|N_{i} N_{f}\right|^{2} \frac{2 \pi \delta\left(E_{f}-E_{i}\right)}{\hbar} \frac{Z^{2} e^{4} \hbar^{4}}{\left|\boldsymbol{p}_{i}-\boldsymbol{p}_{f}\right|^{4}}
$$

Due to normalization of the plane wave function over a box with volume $V$, we have $\left|N_{i} N_{f}\right|^{2}=1 / V^{2}$. The flux factor for a single particle is given by

$$
\text { flux factor }=\left|\boldsymbol{u}_{i}\right| \frac{1}{V}=\left|\boldsymbol{p}_{i}\right| \frac{1}{m V}
$$

while the phase space factor is

$$
\mathrm{d} \text { Lips }=\frac{V}{(2 \pi \hbar)^{3}} \mathrm{~d}^{3} \boldsymbol{p}_{f}
$$

According to the relation (4.33) the cross-section is

$$
\mathrm{d} \sigma=\frac{1}{V^{2}} \frac{2 \pi \delta\left(E_{f}-E_{i}\right)}{\hbar} \frac{Z^{2} e^{4} \hbar^{4}}{\left|\boldsymbol{p}_{i}-\boldsymbol{p}_{f}\right|^{4}} \frac{m V}{\left|\boldsymbol{p}_{i}\right|} \frac{V}{(2 \pi \hbar)^{3}} \mathrm{~d}^{3} \boldsymbol{p}_{f}
$$


Since $\mathrm{d} E_{f}=\mathrm{d}\left(p_{f}^{2} /(2 m)\right)=\left(p_{f} / m\right) \mathrm{d} p_{f},\left|\boldsymbol{p}_{i}\right|=p_{i}=p_{f}$, and $\left|\boldsymbol{p}_{i}-\boldsymbol{p}_{f}\right|^{2}=4 p_{i}^{2} \sin ^{2}(\theta / 2)$ the differential cross-section is:

$$
\begin{aligned}
\frac{\mathrm{d} \sigma}{\mathrm{d} \Omega} & =\frac{Z^{2} e^{4}}{16 p_{i}^{4} \sin ^{4}(\theta / 2)} \frac{m^{2}}{4 \pi^{2}} \int_{-\infty}^{+\infty} \delta\left(E_{f}-E_{i}\right) \mathrm{d} E_{f} \\
& =\frac{Z^{2} e^{4} m^{2}}{64 \pi^{2} p_{i}^{4} \sin ^{4}(\theta / 2)}
\end{aligned}
$$

The kinetic energy of the incoming charged particle is $E_{k i n}=p_{i}^{2} /(2 m)$. We define $a=e^{2} /(4 \pi)$ and therefore the differential cross-section is

$$
\frac{\mathrm{d} \sigma}{\mathrm{d} \Omega}=\frac{Z^{2} a^{2}}{16 E_{k i n}^{2} \sin ^{4}(\theta / 2)}
$$

This is the well-known Rutherford scattering formula.

\subsection{Dirac Equation}

The Dirac equation with small variations, as will be shown below and with the help of what has already been mentioned in this section, is perfectly compatible with the hypothesis of the absolute reference system. We want to find a squared equation, which gives the wave Equation (4.1). This equation has the following form:

$$
\frac{i}{\gamma c} \frac{\partial}{\partial t} \psi=\left(-i \boldsymbol{a} \cdot \nabla+\frac{\beta m c}{\hbar}\right) \psi
$$

This is certainly a well-known problem whose solutions for $\alpha$ and $\beta$ are $4 \times 4$ matrices. Here we choose the Dirac-Pauli representation,

$$
\alpha=\left(\begin{array}{ll}
0 & \sigma \\
\sigma & 0
\end{array}\right) \text { and } \beta=\left(\begin{array}{cc}
\mathbb{1} & 0 \\
0 & -\mathbb{1}
\end{array}\right)
$$

We also define the four components of the matrix $\gamma$,

$$
\gamma=\beta \boldsymbol{\alpha}=\left(\begin{array}{cc}
0 & \boldsymbol{\sigma} \\
-\boldsymbol{\sigma} & 0
\end{array}\right) \quad \text { and } \quad \gamma_{0}=\beta=\left(\begin{array}{cc}
\mathbb{1} & 0 \\
0 & -\mathbb{1}
\end{array}\right)
$$

so the Equation (4.42) becomes,

$$
\frac{i}{\gamma c} \gamma_{0} \frac{\partial \psi}{\partial t}+i \gamma \cdot \nabla \psi-\frac{m c}{\hbar} \psi=0
$$

where we have used the symbol $\gamma$ as the Lorentz contraction coefficient and the symbols $\gamma_{0}$ and $\gamma=\left(\gamma_{1}, \gamma_{2}, \gamma_{3}\right)$, as the mentioned above matrices.

We can use Dirac spinors to write plane wave solutions ${ }^{9}$ of the Equation (4.43). Consider

$$
\psi(\boldsymbol{r}, t)=\left(\begin{array}{c}
\chi(\boldsymbol{p}) \\
\phi(\boldsymbol{p})
\end{array}\right) \mathrm{e}^{i(\boldsymbol{p} \cdot \boldsymbol{r}-2 E t) / \hbar}
$$

where $\chi$ and $\phi$ are two-components spinors.

By substituting this last wave function in the (4.43) equation we get the following solutions:

${ }^{9}$ ref. [11], Thirteenth Lecture, Solution of the Dirac Equation for a Free Particle. 


$$
\begin{aligned}
& \boldsymbol{\sigma} \cdot \boldsymbol{p} \chi=\left(\frac{2 E}{\gamma c}+m c\right) \phi \\
& \boldsymbol{\sigma} \cdot \boldsymbol{p} \phi=\left(\frac{2 E}{\gamma c}-m c\right) \chi
\end{aligned}
$$

The second of the Equations (4.44) gives:

$$
\chi=\frac{\boldsymbol{\sigma} \cdot \boldsymbol{p}}{\frac{2 E}{\gamma c}-m c} \phi=\frac{\gamma}{\gamma-1} \frac{\boldsymbol{\sigma} \cdot \boldsymbol{u}}{c} \phi
$$

where $\gamma=\left(1-\boldsymbol{u}^{2} / c^{2}\right)^{-1 / 2}, \quad \boldsymbol{p}=m \gamma \boldsymbol{u}$. The denominator of the last equation tends to zero when the $|\boldsymbol{u}| / c$ ratio tends to zero, so the fraction tends to infinity. This second solution is therefore not acceptable. In order this equation to be accepted, we change the positive signs of energy and momentum expression to negative, by accepting the Feynman-Stückelberg interpretation. Such a solution will obey the equation:

$$
\frac{i}{\gamma c} \gamma_{0} \frac{\partial \psi}{\partial t}+i \gamma \cdot \nabla \psi+\frac{m c}{\hbar} \psi=0
$$

Since the space of the absolute reference system is the three-dimensional Euclidean space, the four-dimensional space-time will be an extension of this Euclidean space in the four dimensions. In particular, the space-time metric tensor will be the Kronecker $\delta_{i j}$, where $i, j=0,1,2,3$. There is also no difference in using upper and lower indexes, that is for each four-vector $A$ applies $A^{j}=A_{j}$ for $j=0,1,2,3$ and the same applies to each tensor, of any order, in contrast with the covariant and contravariant four-vectors in Minkowski's pseudo-Euclidean space-time. The use of tensor $g_{i j}$, which is the Minkowski space-time metric tensor, is here only used for changing the sign of spatial components of a four-vector in Euclidean space-time. An example is the momentum four-vector of Euclidean space-time $p=(2 E /(\gamma c), \boldsymbol{p})$, in which the momentum vector is reversed according to the relation $g p=(2 E /(\gamma c),-\boldsymbol{p})$.

A very important remark is that the Dirac equation does not need to be relativistically covariant under a Lorentz transformation in the hypothesis of the absolute reference system, since this physics is not relativistic.

Considering all of this, the components of $\gamma$ obey the equation $\gamma^{i}=\gamma_{i}$ for $i=0,1,2,3$. We define the Euclidean space-time four-vectors

$\gamma=\left(\gamma_{0}, \gamma_{1}, \gamma_{2}, \gamma_{3}\right)=\left(\gamma_{0}, \gamma\right), \partial=\left(\frac{1}{\gamma c} \frac{\partial}{\partial t}, \hat{i} \frac{\partial}{\partial x}, \hat{j} \frac{\partial}{\partial y}, \hat{k} \frac{\partial}{\partial z}\right)=\left(\frac{1}{\gamma c} \frac{\partial}{\partial t}, \nabla\right)$, $x=\left(x_{0}, x_{1}, x_{2}, x_{3}\right)=(\gamma c t, r)$ and making use of the Feynman slash notation, we get the inner product

$\nexists=\gamma \partial=\gamma_{i} \partial_{i}=\left(\gamma_{0} \frac{\partial}{\partial x_{0}}+\gamma_{1} \frac{\partial}{\partial x_{1}}+\gamma_{2} \frac{\partial}{\partial x_{2}}+\gamma_{3} \frac{\partial}{\partial x_{3}}\right)=\left(\gamma_{0} \frac{1}{\gamma c} \frac{\partial}{\partial t}+\gamma \cdot \nabla\right)$, where we have introduced the summation convention. The Dirac equation for particles is

$$
\left(i \nexists-\frac{m c}{\hbar}\right) \psi=0
$$


The Dirac equation for anti-particles is

$$
\left(i \nexists+\frac{m c}{\hbar}\right) \psi=0
$$

The plane wave solutions for the particles take the form

$$
\psi_{p}(x)=\mathcal{N}\left(\begin{array}{c}
\chi_{i} \\
\frac{\boldsymbol{\sigma} \cdot \boldsymbol{p}}{2 E}+m c \\
\gamma c
\end{array}\right) \frac{1}{\sqrt{V}} \mathrm{e}^{-i p g x / \hbar}=u_{i}(\boldsymbol{p}) \frac{1}{\sqrt{V}} \mathrm{e}^{-i p g x / \hbar}
$$

where the spinors $\chi_{i}$ for $i=1,2$ are given by

$$
\chi_{1}=\left(\begin{array}{l}
1 \\
0
\end{array}\right), \chi_{2}=\left(\begin{array}{l}
0 \\
1
\end{array}\right)
$$

and $g x=\left(x_{0},-x_{1},-x_{2},-x_{3}\right)=(\gamma c t,-\boldsymbol{r}), \quad p=\left(p_{0}, p_{1}, p_{2}, p_{3}\right)=\left(\frac{2 E}{\gamma c}, \boldsymbol{p}\right)$, $p g x=2 E t-\boldsymbol{p} \cdot \boldsymbol{r}$. For antiparticles,

$$
\psi_{a}(x)=\mathcal{N}\left(\begin{array}{c}
\frac{\boldsymbol{\sigma} \cdot \boldsymbol{p}}{\frac{2 E}{\gamma c}+m c} \chi_{i} \\
\chi_{i}
\end{array}\right) \frac{1}{\sqrt{V}} \mathrm{e}^{i p g x / \hbar}=v_{i}(\boldsymbol{p}) \frac{1}{\sqrt{V}} \mathrm{e}^{i p g x / \hbar}
$$

According to Dirac algebra we have

$$
\begin{aligned}
& \left(\gamma_{0}\right)^{2}=\mathbb{1}_{4 \times 4} \\
& \left(\gamma_{1}\right)^{2}=\left(\gamma_{2}\right)^{2}=\left(\gamma_{3}\right)^{2}=-\mathbb{1}_{4 \times 4} \\
& \gamma_{0} \gamma_{a}=-\gamma_{a} \gamma_{0}, \quad \text { for } a=1,2,3
\end{aligned}
$$

The Hermitian conjugate of $\gamma_{\mu}$ four-vector,

$$
\gamma_{\mu}^{\dagger}=\gamma_{0} \gamma_{\mu} \gamma_{0}
$$

so the Hermitian conjugate of Dirac equation is

$$
i \partial_{\mu} \psi^{\dagger} \gamma_{0} \gamma_{\mu}+m \psi^{\dagger} \gamma_{0}=0
$$

Now we multiply the Dirac equation from the left by $\psi^{\dagger} \gamma_{0}$ and we multiply the Hermitian conjugate of Dirac equation from the right by $\psi$ :

$$
\psi^{\dagger} \gamma_{0}\left(\partial_{\mu} \gamma_{\mu} \psi\right)+\left(\partial_{\mu} \psi^{\dagger} \gamma_{0} \gamma_{\mu}\right) \psi=0
$$

Consequently, we realize that if we define a current as

$$
j_{\mu}=\psi^{\dagger} \gamma_{0} \gamma_{\mu} \psi
$$

then this current satisfies a continuity equation, $\partial_{\mu} j_{\mu}=0$. The first component of this current is simply

$$
j_{0}=\rho=\psi^{\dagger} \psi=\sum_{i=1}^{4}\left|\psi_{i}\right|^{2}
$$

which is always positive.

Substituting the plane wave solution $\psi=u(p) \mathrm{e}^{-i p g x}$, and integrating over a 
volume $V$ we find

$$
\int_{V} \rho \mathrm{d}^{3} x=\int_{V} u^{\dagger}(p) \frac{1}{\sqrt{V}} \mathrm{e}^{i p g x} u(p) \frac{1}{\sqrt{V}} \mathrm{e}^{-i p g x} \mathrm{~d}^{3} x=\mathcal{N}^{2} \frac{\frac{4 E}{\gamma c}}{\frac{2 E}{\gamma c}+m c}
$$

Consequently, in order to have one particle per volume $V$ we choose

$$
\mathcal{N}=\sqrt{\frac{2 E+m \gamma c^{2}}{4 E}}
$$

\section{Conclusions}

Quantum theory, based on the assumption of the absolute reference system, in the context of quantum mechanics and quantum field theory, is simpler than current quantum theory and gives, with minor differences, the same theoretical results. To sum up, the study so far, based on the hypothesis of the absolute reference system, concludes with the following conclusions:

1) The wave behavior of particles based on the classical wave equation, instead of the Schrodinger equation, gives theoretical results in agreement with those of quantum electrodynamics. It is also noteworthy that based on the hypothesis of the absolute reference system, there are no negative energy values such as those derived from the Klein-Gordon equation, and the Dirac theory.

2) The theoretical energy values in the W. Bertozzi experiment (section 1.2) agree with the corresponding experimental ones, in contrast to the theoretical energy values derived from the special theory of relativity for this experiment, which are not in agreement with the experimental results.

3) The dynamics of the hypothesis of the absolute reference system is confirmed experimentally.

\section{Conflicts of Interest}

The author declares no conflicts of interest regarding the publication of this paper.

\section{References}

[1] Patrinos, K. (2019) The Physics of an Absolute Reference System. Journal of Applied Mathematics and Physics, 7, 431-475. https://doi.org/10.4236/jamp.2019.73033

[2] Perkins, D.H. (1987) Introduction to High Energy Physics. Addison-Wesley Publishing Company, Boston.

[3] Bertozzi, W. (1964) Education Development Center, Newton, Mass. 1962 "The Ultimate Speed". American Journal of Physics, 32, 551-555. https://doi.org/10.1119/1.1970770

[4] French, A.P. (1968) Special Relativity. The MIT Introductory Physics Series, W.W. Norton \& Company, New York.

[5] Patrinos, K. (2018) The Physics of an Absolute Reference System. Konstantinos Patrinos, Athens. 
[6] Jackson, J.D. (1975) Classical Electrodynamics. Second Edition, Wiley Eastern Limited, New York.

[7] Born, M. and Wolf, E. (1975) Principles of Optics "Electromagnetic Theory of Propagation, Interference and Diffraction of Light". Fifth Edition, Pergamon Press, Oxford.

[8] Goldstein, H. (1980) Classical Mechanics. Second Edition, Addison-Wesley Publishing Company, Boston.

[9] Ryder, L.H. (1985) Quantum Field Theory. Cambridge University Press, Cambridge.

[10] Landau, L. and Lifshitz, E. (1975) The Classical Theory of Fields. Pergamon Press Ltd., Oxford.

[11] Feynman, R.P. (1961) Quantum Electrodynamics. A Lecture Note and Reprint Volume, W. A. Benjamin, New York. 